\title{
Gait Recognition of Acceleration Sensor for Smart Phone Based on Multiple Classifier Fusion
}

\author{
Zhan Huan (iD, Xuejie Chen, Shiyun Lv, and Hongyang Geng \\ School of Information Science \& Engineering, Changzhou University, Wujin, Jiangsu 213164, China \\ Correspondence should be addressed to Zhan Huan; hzh@cczu.edu.cn
}

Received 8 March 2019; Revised 18 May 2019; Accepted 3 June 2019; Published 20 June 2019

Academic Editor: Roberto Caldelli

Copyright (c) 2019 Zhan Huan et al. This is an open access article distributed under the Creative Commons Attribution License, which permits unrestricted use, distribution, and reproduction in any medium, provided the original work is properly cited.

\begin{abstract}
Gait, as a kind of biological feature, has a profound value in personnel identification. This paper analyzes gait characteristics based on acceleration sensors of smart phones and proposes a new gait recognition method. First, in view of the existing methods in the process of extraction of gait features, a large number of redundant calculations, cycle detection error, and the phase deviation issue during the week put forward the Shape Context (SC) and Linear Time Normalized (LTN) combining SCLTN calibration method of gait cycle sequence matching, to represent the whole extract typical gait cycle gait. In view of the existing extracted gait features are still some conventional features; the velocity change of relatively uniform acceleration and the change of acceleration per unit time are proposed as new features. Secondly, combining new features with traditional features to form a new feature is set for training alternative feature set, from which the training time and recognition effect of multiple classifiers are screened. Finally, a new multiclassifier fusion method, Multiple Scale Voting (MSV), is proposed to fuse the results of Multiple classifiers to obtain the final classification results. In order to verify the performance of the proposed method, gait data of 32 testers are collected. The final experimental results show that the new feature has good separability, and the recognition rate of fusion feature set after MSV algorithm is $98.42 \%$.
\end{abstract}

\section{Introduction}

The human gait has a very unique pattern that can be used for identification and verification. Traditional gait identification that is based on wearable sensors acquires gait data through bundling dedicated sensors on the fixed part of the human body [1]. With the rapid popularization of smartphones and the continuous development of their functions in recent years, it has become possible to use the built-in accelerometer of the smartphone for gait identification for users in conditions that do not affect users' normal work, study, and life [2]. However, factors such as the variety of smartphones and very large differences in performance of the built-in accelerometers lead to significant differences in the collected data, which increases the difficulty of identifying people and directly affects the accuracy and credibility of the recognition.

To improve the accuracy of gait recognition, we conducted continuous research on gait identification based on smartphone accelerometers, and the results are mainly reflected in the following three aspects.
(1) Gait Period Extraction and Feature Selection. Literature [8] proposed a stable periodic detection mechanism, which makes the period detection more "automated." By using this mechanism, the system performance is greatly improved and is more efficient compared to the previous, more complex period detection method. Literature [9] extracted the gait frequency, symmetry coefficient, numerical fluctuation range, and similarity coefficient of the feature curve from the gait data as features. Finally, a weight voting mechanism is proposed according to these features for classification and identification, and experimental results show that these proposed features can be effectively classified and identified.

(2) Improvement and Proposing a New Classification Algorithm. Literature [10] proposed a new dynamic time warping (DTW) algorithm, which uses the time and amplitude of feature points as a two-dimensional sequence. The minimum cumulative distance path is searched by pairs of peaks and troughs, and then the identification is realized by threshold judgement. However, currently, the method has only been 
validated for small samples. Literature [11] proposed a gait period extraction method based on piecewise linear approximation (PLA), a new type of elastic distance metric kernel. A GDTW function is introduced based on the original support vector machine (SVM) Gaussian kernel. Through verifying the performance of the DTW and PLA methods in extracting the gait period in experiments, the results show that the proposed method greatly improves the classification results.

(3) Use Ensemble Learning to Complete Learning Tasks by Building Multiple Learners. Literature [12] used the ant colony algorithm to optimize the multiclassifiers and proposed the high-confidence weighted voting method to fuse classifier outputs, which increases the final model recognition rate by more than 3\% compared to the single classifier. Literature [13] uses the output of multiple speed learning machines to perform a simple mean algorithm fusion processing, and the final model output recognition accuracy is 3.6\% higher than that of a single speed learning machine. The advantage of ensemble learning is the ability to combine multiple learners, and it can often obtain better generalization performance than a single learner. However, it also depends on the selection of a fusion algorithm. The appropriate fusion algorithm often obtains better generalization ability and vice versa.

The above studies develop a universal feature selection for the human acceleration signal and obtain excellent recognition rates, but they do not explore the motion features of the person while walking and only select the single classifier with the best recognition rate to explore. Due to these deficiencies, this paper extracts the rates of acceleration changes and amounts of velocity change associated with relative to uniform acceleration from gait data as new features, combines common time-frequency domain features to model and identify multiple classifiers, and proposes multiscale voting (MSV) for fusion processing to obtain final recognition results.

\section{Related Studies}

Here, we briefly introduce existing methods of gait feature extraction and multiclassifier fusion.

Literature [9] extracted the gait frequency, symmetry coefficient, numerical fluctuation range, and the similarity coefficient of the feature curve from gait data as features, and they proposed a weight voting mechanism for the classification and recognition of gait according to these features.

The specific scheme is as follows.

(1) All the characteristic curves in the database are analyzed one by one to calculate the gait frequency $F_{i}$, symmetry coefficient $S_{i}$, and dynamic range $D_{i}(1 \leq i \leq M)$.

(2) Being calculated from the measured linear acceleration data: these are gait frequency $F$, symmetry coefficient $S$, and dynamic range $D$.

(3) The characteristic curve of linear acceleration is compared with the sample in database and calculate the similarity coefficient $C_{i}$. The result is denoted as $V_{i}^{4}$.

(4) The weighted voting process is as follows.

(a) Calculate the absolute error between $F$ and $F_{i}$. The result is denoted as $V_{i}^{1}$. (b) Calculate the absolute error between $S$ and $S_{i}$. The result is denoted as $V_{i}^{2}$.

(c) Calculate the absolute error between $D$ and $D_{i}$. The result is denoted as $V_{i}^{3}$.

Sum the weighted number of votes for each sample in the database.

$$
V_{i}=w_{1} V_{i}^{1}+w_{2} V_{i}^{2}+w_{3} V_{i}^{3}+w_{4} V_{i}^{4}, \quad i=1,2, \ldots, M
$$

where $W=\left[w_{1}, w_{2}, w_{3}, w_{4}\right]$ is the weighted coefficient vector.

(d) The judgment of the vote: the current measurement data matches the sample with the smallest weighted voting sum in a given database.

The weighted coefficient can be chosen by further experimental data. The similarity coefficient of feature curves is of the most importance for gait identification, followed by gait frequency. The symmetry coefficient has a close relationship with the iPhone's placement. The dynamic range is greatly affected by noise. Therefore, the weighted coefficients are set as $W=\left[2,2,1, w_{4}\right]$ in this paper, where

$$
w_{4}=\left\{\begin{array}{ll}
8 & C_{\max } \in[0.9,1] \\
4 & C_{\max } \in[0.8,0.9) \\
2 & C_{\max } \in[0.7,0.8) \\
1 & C_{\max } \in[0,0.7)
\end{array} \quad C_{\max }=\max _{i \in[1, M]}\left\{C_{i}\right\},\right.
$$

Although the experimental results show that these proposed features can be effectively classified and identified, these types of features are still relatively traditional timefrequency domain features, and motion features of gait have not been studied.

In the multiclassifier fusion algorithm, a threshold of the weighting coefficient is often assigned to the base classifier and the rationality of the threshold allocation directly affects the recognition accuracy of the entire model. Most existing studies use expert experiences as the threshold for assigning weighting coefficients to classifiers, which often leads to inaccurate classifier identification. Reasons may include the following. (1) Experts' occasional mistakes lead to lower recognition accuracy; and (2) unfounded manual intervention in the identification process leads to unrecognized final identification results. Literature [12] proposed a HighReliability Weighted Voting (HRWV) model to address the above problems. The basic idea of high credibility is to use the base classifier's classification recognition credibility as the threshold of its weighting coefficient, which can avoid unreliable human assignment and the influence of human intervention on machine identification, ultimately guaranteeing the threshold reliability and sustainability of the weighting coefficients.

$$
\varphi_{i j}= \begin{cases}\frac{E\left(x_{j}\right)}{E\left(x_{i j}\right)}, & E\left(x_{i j}\right) \geq E\left(x_{j}\right) \\ \frac{E\left(x_{i j}\right)}{E\left(x_{j}\right)}, & E\left(x_{i j}\right)<E\left(x_{j}\right)\end{cases}
$$


where $\varphi_{i j}$ indicates the confidence of the $i$ th base classifier output the $j$ class sample; $E\left(x_{j}\right)$ indicates the actual number of $j$ class samples; $E\left(x_{i j}\right)$ indicates the number of $j$ class samples that the test sample is classified into as class samples after passing the $i$ th base classifier.

$$
\overline{\varphi_{j}}=\frac{1}{n} \sum_{i=1}^{n} \varphi_{i j}
$$

where $\overline{\varphi_{j}}$ represents the average confidence of $n$ numbers base classifier output $j$ class samples.

$$
\varepsilon_{i j}= \begin{cases}\overline{\varphi_{j}}, & \max _{i=1}^{n} \varphi_{i j} \neq \varphi_{i j} \\ 1-\overline{\varphi_{j}}, & \max _{i=1}^{n} \varphi_{i j} \neq \varphi_{i j}\end{cases}
$$

where $\varepsilon_{i j}$ represents the weighting coefficient threshold of the $i$ th base classifier for the $j$ class sample. After the $n$ number classifier classifies $j$ class samples, the classifier threshold with the highest credibility is set to the average credibility, and the weighting coefficient thresholds of the other $n-1$ base classifiers are set to 1-average credibility.

The resulting classifier thresholds and voting methods are combined at the end of the HRWV algorithm. Experimental results show that the recognition rate of the HRWV algorithm is $3 \%$ higher than the single classifier, but the average confidence will be less than 0.5 if most classifiers identify poorly for a certain type of sample, which will result in an increased misclassification of the classifier weighting threshold and the desired fusion effect cannot be achieved.

Therefore, for conditions of gait motion features that are not extracted in the above literature, this paper analyses the motion features of gait by extracting the acceleration rate change and velocity change relative to uniform acceleration. Moreover, the paper proposes a multiscale fusion algorithm for the deficiency of the multiclassifier fusion algorithm.

\section{Data Acquisition and Preprocessing}

To improve the existing gait identification performance based on the smartphone accelerometer, a recognition method based on multiple classifier fusion (MCF) is proposed. The specific algorithm flow is shown in Figure 1.

3.1. Data Acquisition. The traditional method is to fix sensors at positions such as the back of the waist, inner thigh, arm, and ankle to obtain stable gait data. However, when placed on the arm and ankle, there is more noise in the signal due to a series of involuntary jitters of the hands and feet, which affects the integrity and stability of the acquired data. To increase the stability of gait data signal acquisition, the paper places the smartphone in the trouser pocket at the position of inner thigh while collecting data, and the built-in accelerometer is used to collect the data (the specific placement is shown in Figure 2). The Samsung Note II series smartphone is used for acquisition, and the sampling frequency is $100 \mathrm{~Hz}$.

3.2. Data Preprocessing. The original acceleration signal contains high-frequency noise during personnel movement, which mainly originates from two areas: one is the noise generated by the mobile phone accelerometer, and the other is the noise generated by the physiological vibration of the human body during the process of collecting gait data. Considering the high sensitivity of acceleration sensors, even small jitters will affect the change in the acceleration value, which will further affect subsequent feature extraction and classification recognition rate. Therefore, while retaining useful information, trying to remove as much noise interference in the data as possible is also necessary. For the condition of a gait feature signal mainly concentrated in the lowerfrequency part of the data, the third-order Butterworth lowpass filter [14] is used for filtering to remove high-frequency noise. Figure 3 shows the gait data before and after filtering. It has smooth feature of the passband's frequency response curve, which can be expressed by

$$
|H(w)|^{2}=\frac{1}{1+\left(w / w_{c}\right)^{2 n}}=\frac{1}{1+\varepsilon^{2}\left(w / w_{p}\right)^{2}}
$$

\section{Period Division and Feature Extraction}

4.1. Period Division. There are two commonly used period division methods: frame-based segmentation $[15,16]$ and period-based segmentation. Frame-based segmentation is also called segmentation based on a fixed sliding window, where the window represents the amount of data used for processing at one time. Hence, the method divides the period according to the fixed data length and can quickly realize the segmentation process of the time series. The window is also divided into overlapping and nonoverlapping windows, which are mainly determined by the window size and sliding step size. This method is often used in current research because of its simple implementation.

Although the method has excellent real-time performance and can maintain pattern features of the gait, it does not best-reflect the phase of gait and solve time variation problems caused by the gait mode. Unlike frame-based segmentation, the period-based segmentation method is based on the gait period and can fully reflect the hidden biometrics of gait data. Considering that gait acceleration data usually appears as a set of data with periodic features, this paper uses a period-based segmentation method, and the specific algorithm is divided into the following three steps.

(1) Estimation of Period Length. According to statistics, normal people walk two steps on average from $0.8 \mathrm{~s}$ to 1.2 s. The two steps correspond to one period in the data since only one mobile phone is placed in the right side of the pants pocket when collecting the data. In addition, the sampling frequency is set as $100 \mathrm{~Hz}$, such that approximately 80 to 120 data points correspond to one period.

(2) Period Detection. Gait data are periodically detected using the local minimum value [17], which means that data contained between two adjacent minimum values are considered to be a single gait period. 


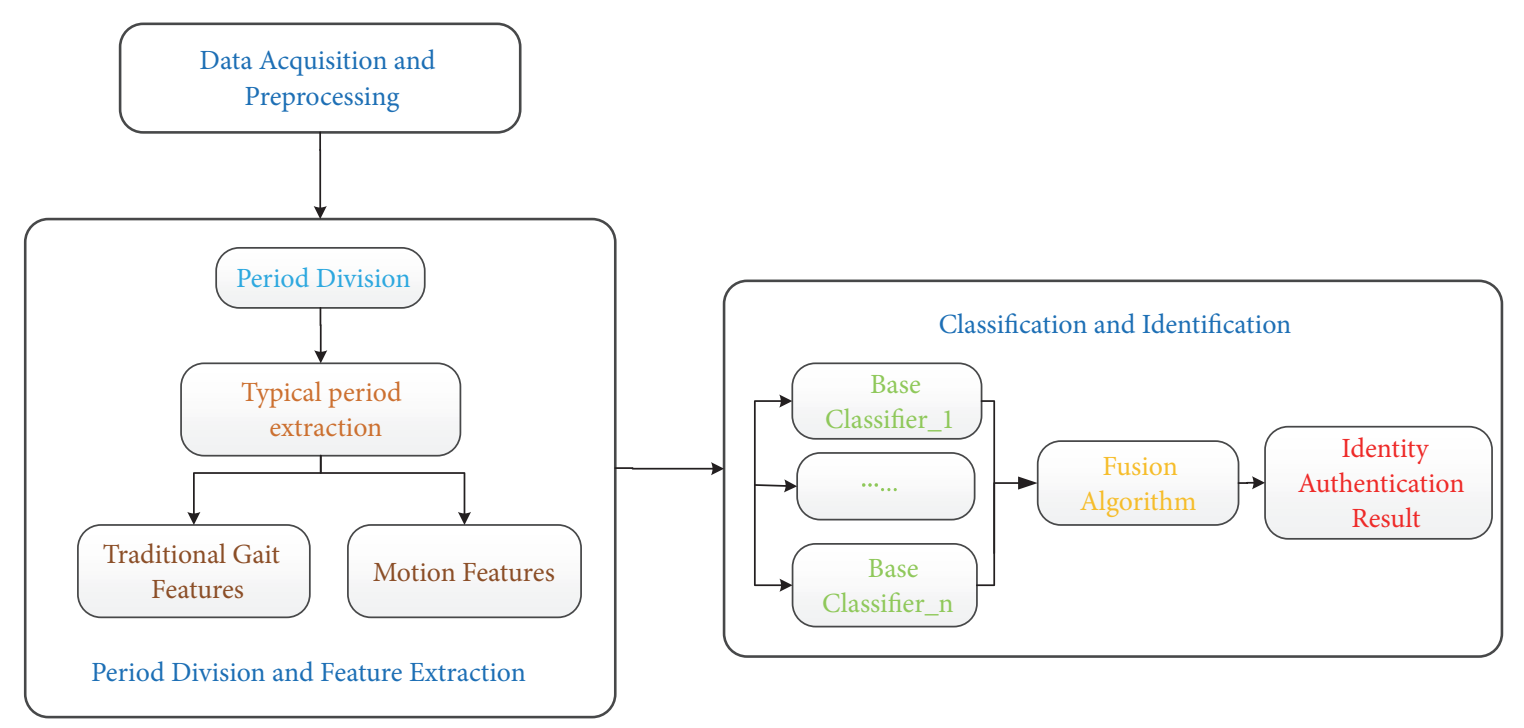

FIGURE 1: Multiple-classifier recognition fusion algorithm.

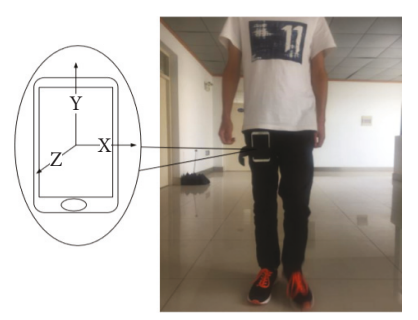

FIgURE 2: Mobile phone location.

(3) Eliminate the Abnormal Period. Gait data can be divided into a single gait period through the two steps mentioned above, but these periods are not all suitable to feature extraction. The abnormal period should be eliminated after filtering these periods. This paper uses DTW to calculate similar distances in the normal period, and periods with large differences in distance are eliminated.

A single gait period of different individuals is extracted through the above steps as shown in Figure 4.

4.2. Typical Period Extraction. Through periodic extraction, a complete set of gait acceleration data can be segmented into multiple small gait periods. Considering periodic features of gait acceleration data, the paper extracts a typical gait period from multiple gait periods to characterize the entire gait data feature to avoid excessive redundant data, which are not conducive to feature extraction. Currently, DTW [18] (and the deformation algorithm derived from the DTW algorithm [19-21]) and linear time normalization (LTN) based on linear matching [22, 23] are often used in a typical period extraction. However, DTW is a nonlinear matching regularization algorithm, which is suitable for decomposing the irregularity of action time relative to the overall action time, especially in the field of speech recognition. However, considering that gait is linear in the period, DTW is not suitable to gait-typical period extraction; in contrast, LTN considers the linear matching relationship between data in the processing of gait period sequencematching before calculating the shortest distance between the two. The matching method of LTN pays more attention to gait linear features compared to DTW, but LTN also has some shortcomings. When matching sequences, shape features of the sequence itself are not taken into account.

For local shape information for each point context that was ignored in the LTN algorithm, the paper introduces the concept of Shape Context (SCLTN) [24-28] in LTN, which allows for the determination of correspondence between each data point in the periodic sequence. The shape context is a description for the shape, whose main purpose is to capture the relative position of each data point in space to achieve a stricter and more accurate match between points, as shown in Figure 5.

First, the linear correspondence between points on two sets of sequences is calculated using LTN, from which the point matching relationship of Figure 5(a) is obtained. Then, the shape context histogram of each matching data point is calculated using the shape context, and the point matching relationship of LTN is verified again to accurately match each point on two sets of sequences. Specific steps for using the SCLTN algorithm to extract typical periods are as follows.

(1) First, calculate the shape context information of each data point in all periods.

(2) Then, calculate the $\mathrm{C}$ value between each data point, finding the optimal alignment of each point in the sequence to ensure that the total $\mathrm{C}$ value is the smallest.

(3) For the alignment sequence obtained in step (2), the linear rule in the LTN algorithm is used again to verify the linear relationship between matching alignment points to ensure that the best matching alignment is achieved.

(4) The linear rule combination in the above SC and LTN is only used to align data points; distances between periods 


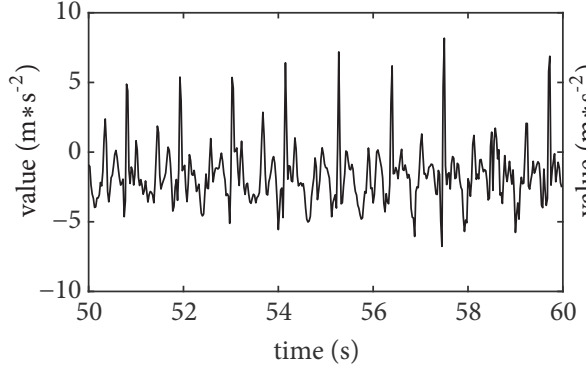

- X axis

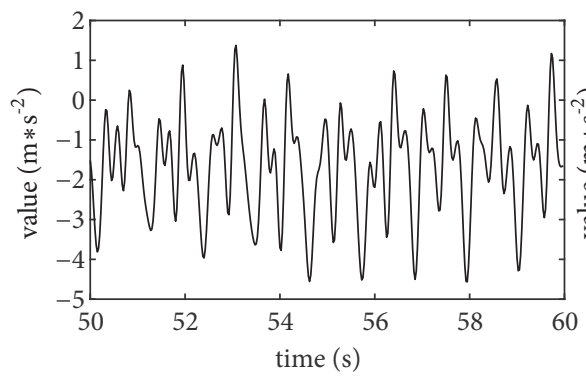

- X axis

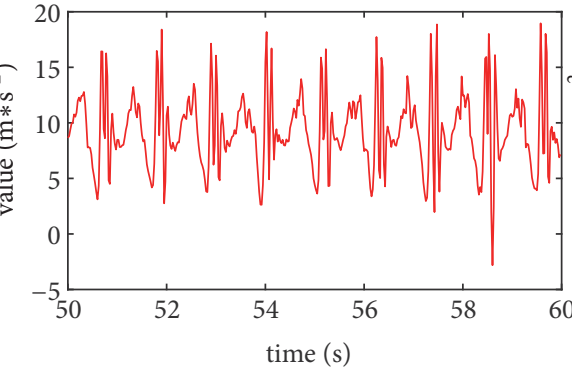

Y $\mathrm{Y}$ axis

(a) Raw triaxial acceleration data
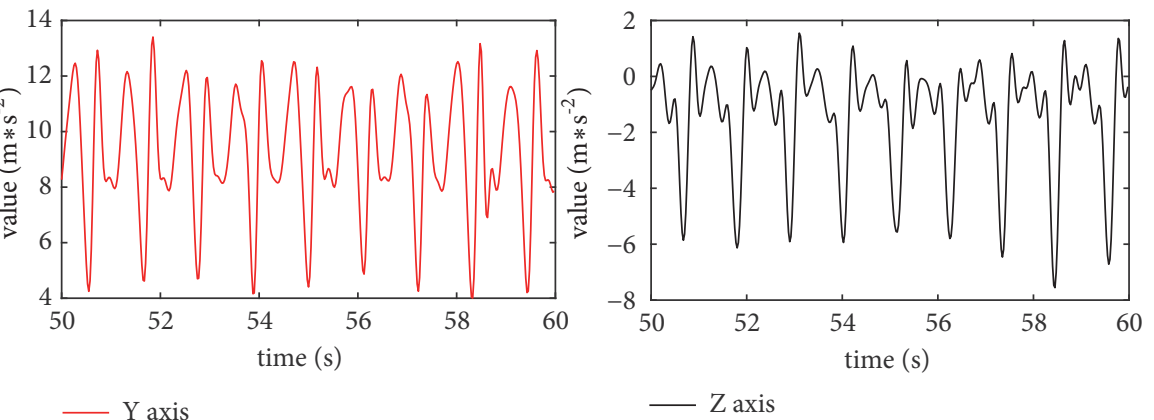

(b) Filtered triaxial acceleration data

FIGURE 3: Data before and after filtering.

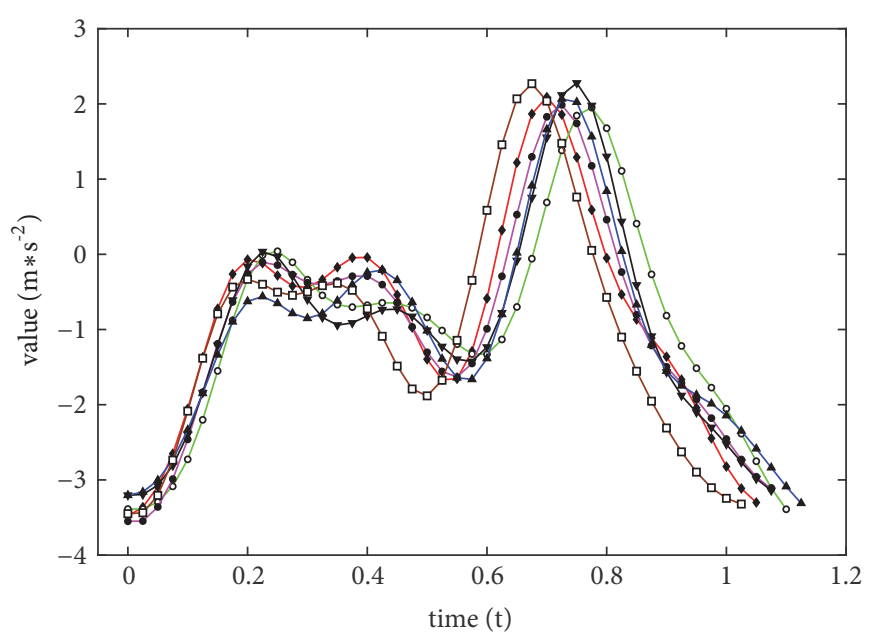

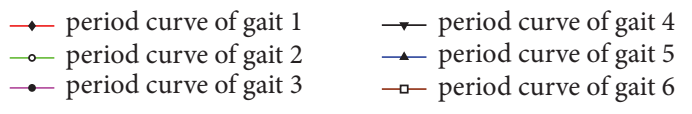

(a) Gait period extracted by individual A

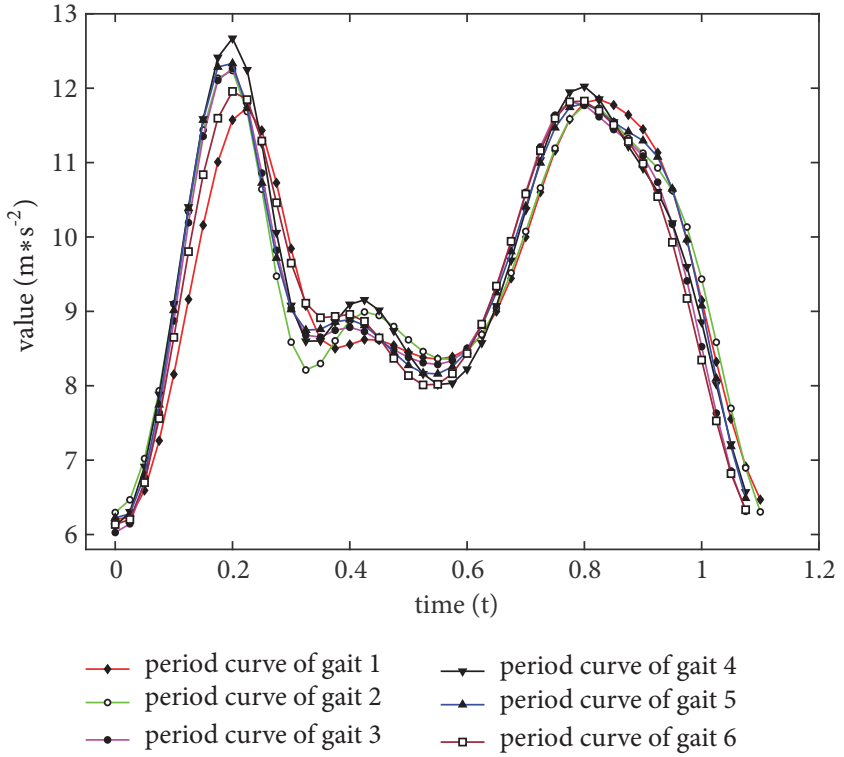

(b) Gait period extracted by individual B

FIGURE 4: The gait period of different individuals.

should still be calculated using the LTN algorithm, and the period corresponding to the minimum distance is selected as the typical period.

This paper does not use the shape context algorithm to determine the final distance between two sets of sequences but makes further improvements of matching points through the shape context. When extracting the typical period, the LTN algorithm is still used to calculate the sequence between the two groups, and the period is extracted according to the distance. The shorter the distance between sequences is, the more similar the two groups are; the greater the distance is, the greater their difference is. 


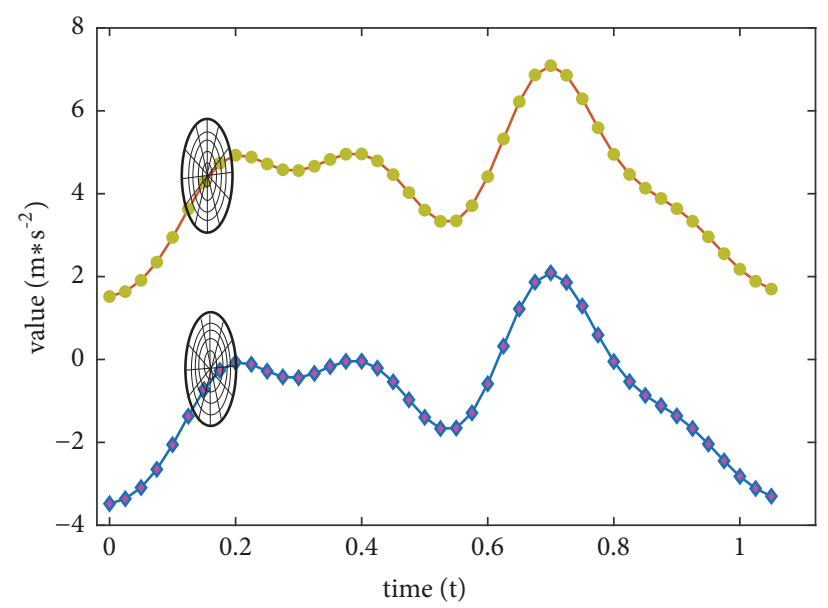

(a) Point matching by using SC-LTN
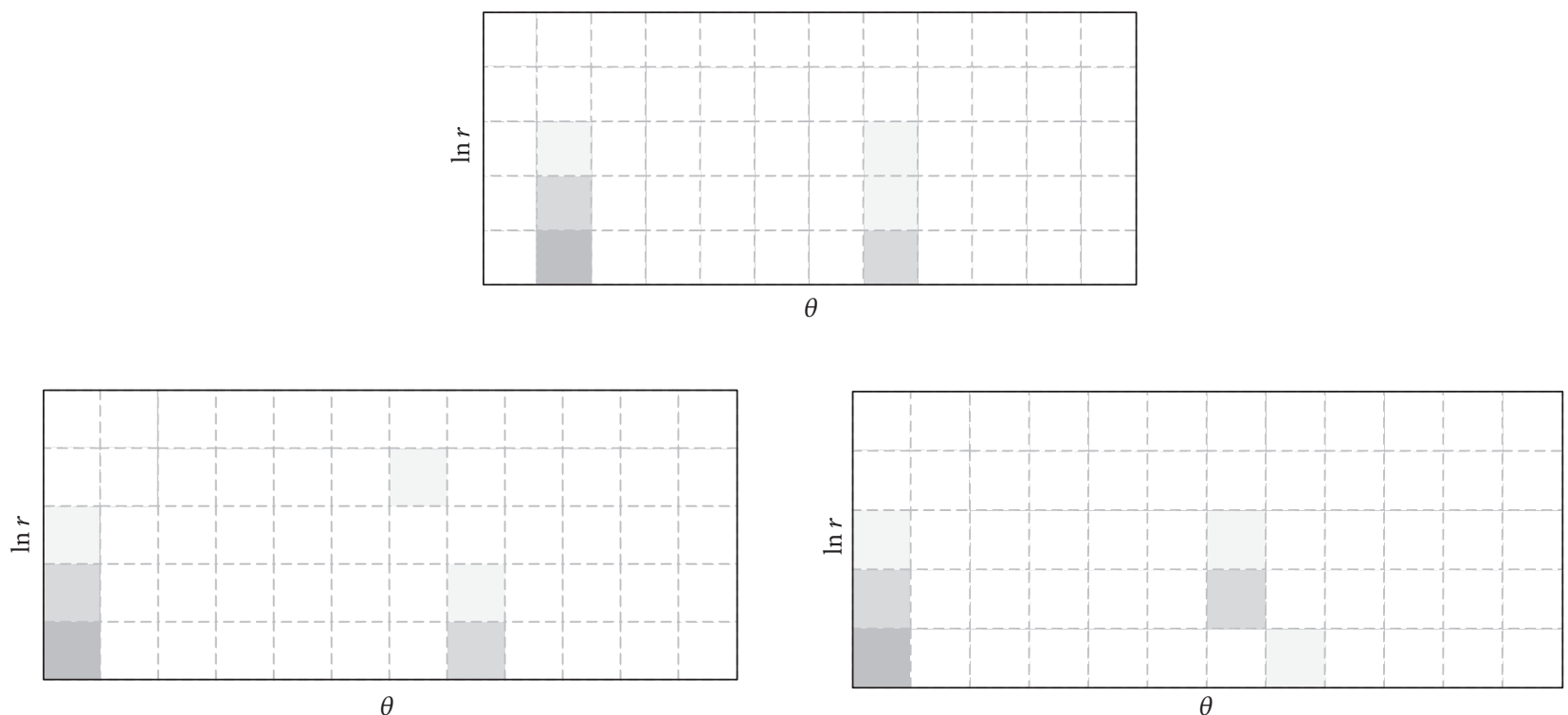

(b) Shape context histogram

FIGURE 5: SC-LTN matching process.

\section{Feature Extraction}

Feature extraction is used to extract feature vectors that can represent individual identities from the obtained single gait period, which is the key in classification recognition technology. The effect of feature extraction will directly affect the accuracy of classifier training and final classification recognition.

5.1. Traditional Gait Features. Traditional methods based on acceleration signal feature extraction are time domain analysis [29], frequency domain analysis [30], and timefrequency domain analysis [31]. Traditional feature values that are extracted in combination with the above methods are shown in Table 1.

A series of time domain features such as average, standard deviation, and zero-crossing can be used to represent waveform features of gait data signals and simple statistical features. A series of features such as steps and stride can be used to represent an individual's biometrics. Frequency domain features such as Fourier coefficients are commonly used to represent periodic information in an individual's gait. Although the traditional feature calculation is simple, few features can represent gait movement of an individual, and the recognition rate obtained using traditional features is not high.

5.2. Motion Features. Since traditional features cannot best reflect biological features of gait, this paper proposes a new gait feature extraction method for extracting individual motion features.

5.2.1. Gait Motion Process Analysis. The motion law of gait can be described by some kinematic parameters, and the 
TABLE 1: Traditional gait features (suppose that the sample of a set of gait data is expressed as $\mathrm{X}=\left\{x_{1}, x_{2}, \ldots, x_{n}\right\}$; $n$ represents the number of samples and $x_{i}$ represents the $i$ th data point).

\begin{tabular}{|c|c|}
\hline Feature name & Formula \\
\hline Average & $\bar{x}=\frac{x_{1}+x_{2}+\cdots+x_{n}}{n}=\frac{1}{n} \sum_{i=1}^{n} x_{i}$ \\
\hline Standard deviation & $\sigma=\sqrt{\frac{1}{n} \sum_{i=1}^{n}\left(x_{i}-\bar{x}\right)^{2}}$ \\
\hline Mean variation & $m=\frac{1}{n} \sum_{i=1}^{n}\left|x_{i}-\bar{x}\right|$ \\
\hline Peak interval & $\begin{array}{c}T_{\min }=\left(t_{x_{i+1}^{\min }}-t_{x_{i}^{\min }}\right) \\
\text { or } T_{\max }=\left(t_{x_{i+1}^{\max }}-t_{x_{i}^{\max }}\right)\end{array}$ \\
\hline energy & $E=\sum_{i=1}^{n}\left|x_{i}\right|^{2}$ \\
\hline Zero-crossing & above_zero $=\sum_{i=1}^{n} \Pi\left(x_{i}>\right.$ zero $)$ \\
\hline Skewness & $S=\frac{1}{n} \sum_{i=1}^{n}\left(\frac{x_{i}-\bar{x}}{\widehat{\sigma}}\right)^{3}, \hat{\sigma}=\sqrt{\frac{1}{n-1} \sum_{i=1}^{n}\left(x_{i}-\bar{x}\right)^{2}}$ \\
\hline Kurtosis & $K=\frac{1}{n} \sum_{i=1}^{n}\left(\frac{x_{i}-\bar{x}}{\widehat{\sigma}}\right)^{4}$ \\
\hline Root mean square & $x_{r m s}=\sqrt{\frac{x_{1}^{2}+x_{2}^{2}+\cdots+x_{n}^{2}}{n}}=\sqrt{\frac{1}{n} \sum_{i=1}^{n} x_{i}^{2}}$ \\
\hline DFT coefficient & $X_{q}=\sum_{j=1}^{n} x_{j} e^{-i(2 \pi / n) j q}, \forall q \in[1, n]$ \\
\hline DCT coefficient & $X_{q}=\frac{1}{2} x_{1}+\sum_{j=2}^{n} x_{j} \cos \left[\frac{\pi}{n} j\left(q+\frac{1}{2}\right)\right], \forall q \in[1, n]$ \\
\hline
\end{tabular}

whole gait movement process can be divided into swing and stance phases.

As shown in Figure 6, the swing stage accounts for about $38 \%$ of the entire gait cycle. First, the swing begins, then it reaches the mid-swing stage through accelerating, and finally it slows down to the terminal-swing stage.

As shown in Figure 7, the standing stage accounts for $62 \%$ of the entire gait cycle, and it mainly consists of the following five parts. The first is the initial-stance stage, which refers to the period after the heel touches the ground and before the forefoot touches the ground. The second is the support reaction stage, which refers to the period after the foot begins to touch the ground and before shifting the weight to another supported foot. The third is the mid-stance stage, which refers to the period after the weight on the supporting foot and before the tiptoes leave the ground. The fourth is the terminal-stance, which refers to the period after the heels leave the ground and before the tiptoes leave the ground. The fifth is the preswing stage, which refers to period when feet successfully leave the ground, and this stage often is referred to as the push-off stage.

Combined with Figures 6 and 7, it can be concluded that the collected gait data are repeated during the standing period and the swing period, so the data collected under ideal conditions should have strict periodicity. However, due to many problems such as sampling equipment and environment, the collected gait data presents the features of quasiperiodicity. Although it loses perfect periodicity, it also retains more uniqueness for the individual, and individual features can be excavated from more data.

5.2.2. Gait Mechanics Analysis. Studies in a large number of literatures have shown that the gravity of the body and the inertial force while walking are put on the foot that touches the ground. When one foot is about to leave the ground and another foot is about to touch the ground, the foot not only will receive gravity but also will be subjected to downward inertial force.

Therefore, the pressure on the foot is maximum at this time. And then the individual will have the force of the acceleration required for the upward movement, so that the other foot can be lifted up and swing successfully. When the individual gait is about to enter the swing stage, the force of the ankle to backward push the ground will gradually become larger, but, during the swinging stage, the ankle will only be subjected to the inertial force generated from backward pushing the ground. Hence, it can be considered as the inertial force when the ankle backward pushing the ground makes the gait in the swing stage, so the swing of the individual legs after the foot leaving the ground is caused by the inertial force. However, since individually oscillating legs are less stressed at the joints, the individual's acceleration while walking is mainly relying on the opposite reaction of the ground to the foot and also gives the individual 


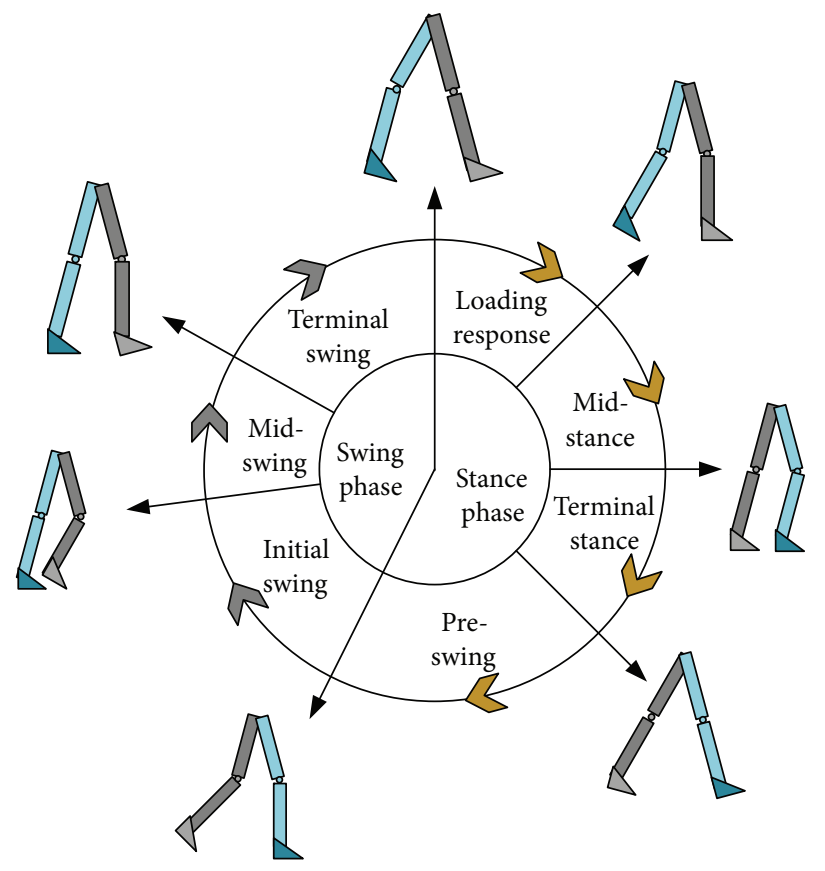

FIgURE 6: Gait period division.

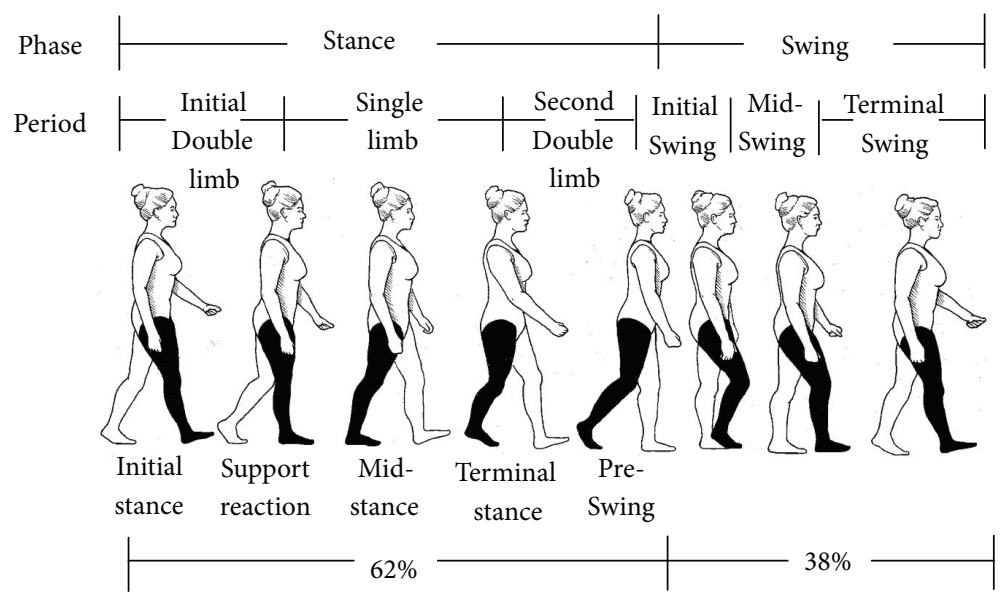

FIGURE 7: Gait stage division of gait.

the force to move forward. But there is no fixed law in the horizontal direction of the individual, and it sometimes becomes positive and sometimes becomes negative. The individual's gait is the continuous movement of one leg to support the body and another leg to repeat swing, which is a process of constantly losing balance and finding balance. Loop back and forth, and therefore most of the individual's muscles appear relaxed during the walk.

Combined with the above discussion and Biomechanical principle of walking from Qian Jingguang et al. [32], it is concluded that the contact force of the foot has an extreme value at the turning point of each gait cycle, and there is a maximum value when the heel touches the ground. When the foot gradually flattens and the force area gradually increases, the force will reduce and reach a minimized value when the foot is fully flattened. Another maximum value will appear when the heel leaves the ground and toes are squatting, in which the force curve has typical symmetrical bimodal properties throughout the gait cycle. And the study suggests that there is no significant difference in the contact force of the human body at different ages, which has a certain stability.

5.2.3. Motion Feature Extraction. Combined with the analysis of gait mechanics in Section 5.2.2, it is concluded that the force curve in the whole gait cycle has typical symmetrical bimodal properties; it can be known from Newton's second law: $F=m \times a$ (where $F$ represents the resultant force of the individual; $m$ represents the individual's own quality; and $a$ represents the individual's acceleration value), so 
the individual's acceleration curve should also exhibit a bimodal feature. In combination with the above analysis, any individual will have two maximum values of acceleration in a single gait cycle. Therefore, the two maximum values can be used to divide the entire gait interval into multiple motion regions and then propose motion features one by one for a single motion area.

Combining the above analysis, the paper, respectively, extracts six features from the single gait cycle: cycle start point $(s t a r t)$, first maximum point $\left(\max _{f}\right)$, first minimum value $\left(\min _{f}\right)$, last minimum value $\left(\min _{e}\right)$, last maximum value $\left(\max _{e}\right)$, and gait cycle end point (end) using the 6 feature points to divide the individual gait data into 5 different motion intervals. The results of the interval division of different individuals according to this method are shown in Figure 8. The connection between two feature points is regarded as a uniform acceleration motion, such that the amount of acceleration change that changes and decelerates per unit time can be expressed as the slope of the five straight lines. The velocity variation of a relatively uniform acceleration motion in a single interval can be represented by the area enclosed by the original variable acceleration curve and the uniform acceleration line. Therefore, this paper provides 16 eigenvalues, namely, 6 eigenvalue points, 5 slopes, and 5 areas.

\section{Classifier Fusion Algorithm}

Single classifier usually only has a high recognition rate on particular individuals; hence, to achieve complementary advantages among various classifiers, the final recognition result is obtained in this paper using a fusion algorithm on the multiclassifier's output. The fusion algorithm plays a crucial role in the multiclassifier fusion model, and its quality directly determines the final recognition rate. The traditional voting method [33] is one of the most commonly used fusion algorithms, whose basic voting rule is that each classifier has voting rights and the arbitration principle is that the minority obeys the majority. However, due to the different performances of each classifier and large differences in the recognition effect for each person, it is difficult for the traditional voting method to combine advantages of multiple classifiers and achieve the desired recognition effect.

For shortcomings of the traditional voting method, this paper proposes a multiscale voting method (MSV) by combining the principles of the voting method with its arbitration principle. The basic idea of MSV algorithm is as follows. When the multiclassifier cannot judge a sample category accurately using the voting method, an unknown sample should classify samples by combining different measures (such as recognition rate of each classifier for its output category and F1_score). The MSV algorithm not only retains the features of traditional voting methods but also combines them with a single classifier for the recognition performance of different types of samples, which makes the advantages of each classifier complementary. The MSV algorithm is mainly divided into two steps: extracting discriminants and constructing voting functions.
6.1. Extracting Discriminants. For a pattern recognition problem with $M$ numbers classifiers $\left(C_{m}, m=1,2, \ldots, M\right)$ and $N$ numbers categories $\left(c_{i}, i=1,2, \ldots, N\right)$, the traditional voting method is used to count repetitions in the number of sample labels output by each classifier and uses the category label with the most repetition as the final classification result. The traditional voting method is advantageous because the algorithm is simple to implement, and the accuracy of recognition is higher when the repetition numbers of different labels are larger. However, the traditional voting method loses the fusion effect on multiple classifiers and does not give accurate classification results when the repetition numbers of the sample labels are extremely similar or equal. Hence, it is necessary to use discriminants to select different criteria. The extraction of discriminants is divided into the following two steps.

(1) According to the following formula, count number $b_{i}\left(b_{i} \in \boldsymbol{B}\right)$ of sample labels output by the $i$ th classifier:

$$
b_{i}= \begin{cases}c_{i}+1 & a_{i}=a_{j}, 1 \leq i \leq M, 1 \leq j \leq M \\ c_{i} & a_{i}=a_{j}, 1 \leq i \leq M, 1 \leq j \leq M\end{cases}
$$

In the formula, $a_{i}$ represents the sample label output by the $i$ th classifier, and the initial value of $c_{i}$ is 1 .

(2) According to the following formula, extract the discriminant $K$ :

$$
\begin{aligned}
& \boldsymbol{K} \\
& = \begin{cases}{[1,0,0,0]} & \max (\boldsymbol{B})>\frac{M}{2} \text { and } \max (\boldsymbol{B})-\min (\boldsymbol{B})>1 \\
{[0,1,0,0]} & 1<\max (\boldsymbol{B}) \leq \frac{M}{2} \\
{[0,0,1,0]} & \max (\boldsymbol{B})=1 \\
{[0,0,0,1]} & \max (\boldsymbol{B})>\frac{M}{2} \text { and } \max (\boldsymbol{B})-\min (\boldsymbol{B}) \leq 1\end{cases}
\end{aligned}
$$

6.2. Constructing Voting Functions. To fully integrate the advantages of each classifier, this paper constructs voting functions $\left(\mathrm{Bel}_{i} \in \boldsymbol{B e l}, 0 \leq i \leq 3\right)$ by combining different discriminants, and, in total, four different voting functions are constructed. The classification effect for any single classifier $C_{m}(m=1,2, \ldots, M)$ can be expressed by the confusion matrix $(C M)$ of

$$
\boldsymbol{C M}_{m}=\left[\begin{array}{ccccc}
n_{11}^{m} & n_{12}^{m} & \cdots & n_{1 N-1}^{m} & n_{1 N}^{m} \\
n_{21}^{m} & n_{22}^{m} & \cdots & n_{2 N-1}^{m} & n_{2 N}^{m} \\
\vdots & \vdots & \ddots & \vdots & \vdots \\
n_{N 1}^{m} & n_{N 2}^{m} & \cdots & n_{N N-1}^{m} & n_{N N}^{m}
\end{array}\right], \begin{aligned}
& \\
& \quad m=1,2, \ldots, M
\end{aligned}
$$

where $n_{i j}^{m}$ indicates that the $m$ th classifier classifies samples of the $c_{i}$ class into number of classes.

Based on the confusion matrix for an unknown sample $x$ that originally belongs to class $c_{i}$, the probability that the $C_{m}$ 


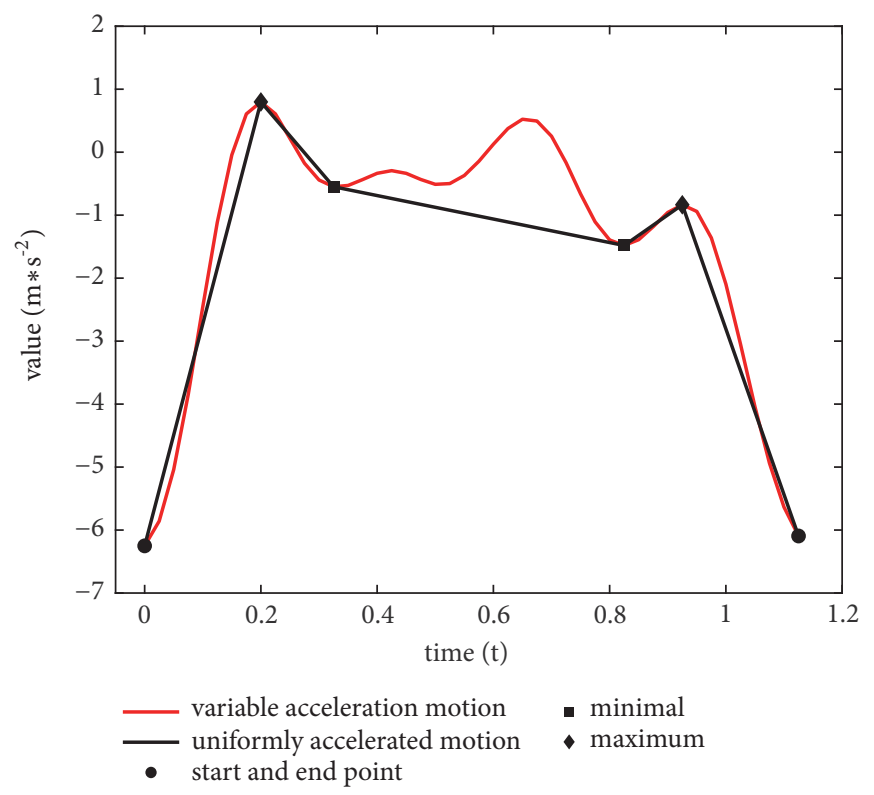

(a) Gait period extracted by individual A

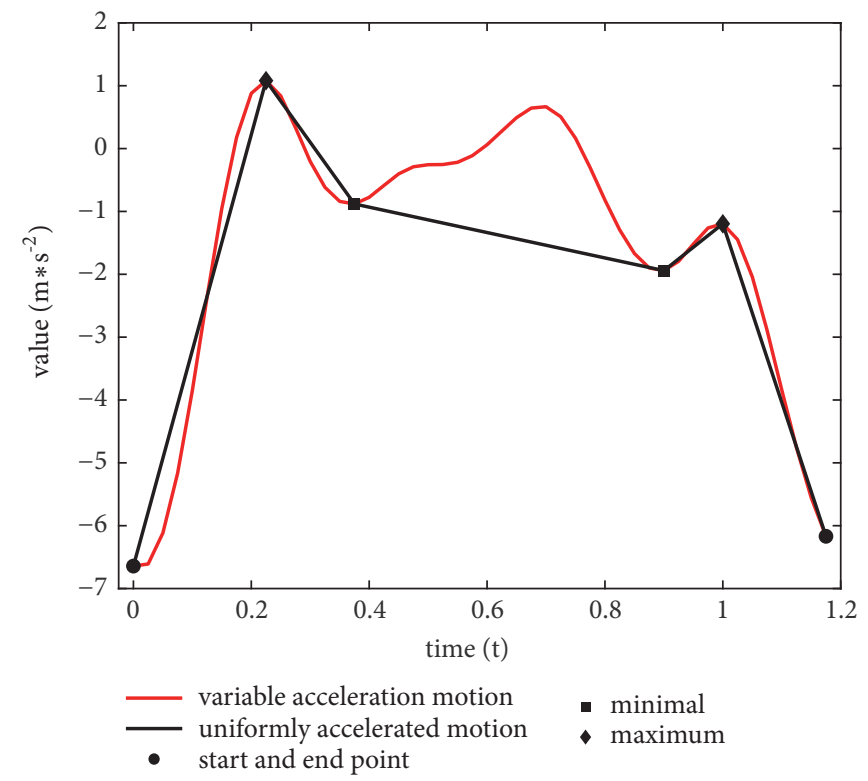

(b) Gait period extracted by individual B

FIGURE 8: Interval division of different individuals.

classifier classifies it into $c_{j}\left(p_{m} \in \boldsymbol{P}\right)$ and the recall rate of class $c_{i}\left(R_{m} \in \boldsymbol{R}\right)$ can be, respectively, calculated using

$$
\begin{aligned}
p_{m}\left(x \in c_{i} \mid C_{m}(x)=c_{j}\right) & =\frac{n_{i j}^{m}}{n_{j}^{m}}=\frac{n_{i j}^{m}}{\sum_{i=1}^{i=M} n_{i j}^{m}} \\
i, j & =1,2, \ldots, N, 1 \leq m \leq M \\
R_{m}\left(x \in c_{i} \mid C_{m}(x)=c_{i}\right) & =\frac{n_{i i}^{m}}{n_{i}^{m}}=\frac{n_{i i}^{m}}{\sum_{j=1}^{j=M} n_{i j}^{m}} \\
i, j & =1,2, \ldots, N, 1 \leq m \leq M
\end{aligned}
$$

According to (10) and (11), the following equation can be derived to calculate the $C_{m}$ classifier's F1_Score $\left(F_{m} \in \boldsymbol{F}\right)$ for class $c_{i}$ :

$$
\begin{aligned}
F_{m}(i, i)=\frac{2 * p_{m}(i, i) * R_{m}(i, i)}{p_{m}(i, i)+R_{m}(i, i)} & \\
& i=1,2, \ldots, N, 1 \leq m \leq M
\end{aligned}
$$

According to (7), (10), (11), and (12), decision weight assignments and voting functions can be, respectively, constructed as follows:

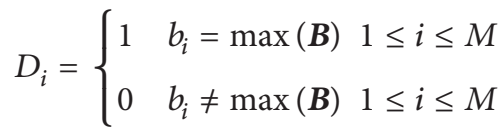

$$
\begin{aligned}
& \operatorname{Bel}_{0}(j)=\frac{\sum_{j=0}^{M} a_{j} * D_{j}}{\sum_{j=0}^{M} D_{j}} \quad 1 \leq \mathrm{j} \leq M
\end{aligned}
$$

Equations (13) and (14) are, respectively, the first voting weight distribution and voting function;

$$
\begin{aligned}
D P_{m} & = \begin{cases}1 & p_{m}=\max (\boldsymbol{P}), 1 \leq m \leq M \\
0 & p_{m}=\max (\boldsymbol{P}), 1 \leq m \leq M\end{cases} \\
\operatorname{Bel}_{1}(j) & =\sum_{j=0}^{M} a_{j} * D P_{j}
\end{aligned}
$$

Equations (15) and (16) are, respectively, the second voting weight distribution and voting functions;

$$
\begin{array}{r}
\operatorname{Bel}_{2}(j) \\
=\max _{i}\left(\frac{\prod_{m=1}^{M} p\left(x \in c_{i} \mid C_{m}(x)=C_{m j}\right)}{\sum_{i=1}^{N} \prod_{m=1}^{M} p\left(x \in c_{i} \mid C_{m}(x)=C_{m j}\right)}\right) \\
i=1,2, \ldots, N
\end{array}
$$

Equation (17) is the third voting function constructed by integrating the recognition results of all classifiers.

$$
\begin{aligned}
& D F_{m}= \begin{cases}1 & F_{m}=\max (\boldsymbol{F}), 1 \leq m \leq M \\
0 & F_{m}=\max (\boldsymbol{F}), 1 \leq m \leq M\end{cases} \\
& \operatorname{Bel}_{3}(j)=\sum_{j=0}^{M} a_{j} * D F_{j}
\end{aligned}
$$

Equations (18) and (19) are, respectively, the fourth voting weight assignment and voting function

For an unknown sample $x$, it is first classified by a multiclassifier, and then the multiclassifier classification result 
is subjected to MSV fusion algorithm to obtain the final classification, which can be calculated using

$$
\text { label }=\boldsymbol{K}^{T} \cdot \mathbf{B e l}
$$

\section{Experimental Results and Analysis}

\subsection{Experimental Preparation}

7.1.1. Experimental Data and Environment. This paper uses data collection methods that were introduced in the data collection section to collect data from 32 volunteers who were approximately 25 years old. They included 16 boys and 16 girls whose heights range from $150 \mathrm{~cm}$ to $190 \mathrm{~cm}$. The volunteers walked on level ground with their walking status for approximately $100 \mathrm{~m}$ during data collection. Each volunteer needs to have 30 sets of independent experimental data collected to avoid contingency of overall experimental data and to ensure that data can better characterize the gait features of the individual.

Experimental environment: this article uses Python 3.6 as the software platform and an Intel Core i7 processor as the hardware platform, with a main frequency of $2.7 \mathrm{GHz}$ and a memory of $8 \mathrm{~GB}$. The 64 -bit Windows 10 operating system is adopted.

7.1.2. Multiple Classifier Selections. Base classifier selection is the basis of multiclassifier fusion to select excellent classifiers for as much fusion as possible, and this paper selects the ten commonly used classifiers as alternatives, shown in Table 2.

7.2. Experimental Design and Analysis of Results. To test the performance of traditional features of data collected in this paper and to verify new feature separability, the fusion of new features and traditional features, and the performance of MSV multiclassifier fusion algorithm, the following four experiments are designed.

(1) Verify the SCLTN method.

(2) Implement multiclassifier screening.

The purpose of multiclassifier screening is to select the most suitable classifier among eight alternative classifiers for fusion. To complete the screening of multiclassifiers, this paper mainly demonstrates the method through following three, small experiments.

(a) Verify that the acquired data have excellent separability according to traditional features and select the appropriate classifier from eight potential base classifiers for fusion.

(b) Classify new features using eight alternative base classifiers and look for alternative classifiers that are suitable for fusion, comparing them to traditional classifiers that are selected by features.

(c) Compare the ability to distinguish samples between new features and traditional features, fusing and classifying new features and traditional features, selecting the final suitable classifier from candidate classifiers, and determining the fusion classifier after comparing with classifiers selected by the former two ones.
TABLE 2: Alternative classifiers.

\begin{tabular}{lc}
\hline Classifier name & Code \\
\hline Bernoulli Naive Bayes & $\mathrm{C} 1$ \\
Gaussian Naive Bayes & $\mathrm{C} 2$ \\
k-Nearest Neighbor & $\mathrm{C} 3$ \\
Ridge Regression & $\mathrm{C} 4$ \\
Decision Tree & $\mathrm{C} 5$ \\
Random Forest & $\mathrm{C} 6$ \\
Support Vector Machine & $\mathrm{C} 7$ \\
Multilayer Perceptron & $\mathrm{C} 8$ \\
\hline
\end{tabular}

(3) Verify the reliability of the MSV fusion algorithm through experimental comparison.

To better train and judge each classifier and to obtain prior knowledge for the MSV fusion algorithm such as the recognition rate of the classifier for a single individual and recall rate, this paper divides the experimental data into 10 equal parts in all classification experiments. Six parts were used as training sets, and the classifier was trained using a ten-fold method during training; two parts were used as test sets to test the classifiers and obtain prior knowledge; and two parts were used as a verification set, mainly verifying the reliability of algorithm and the effect of the fusion algorithm.

(4) Compare with existing research.

The method of this paper is compared with related research in recent years to further reflect the superiority of the identification method adopted in this paper.

7.2.1. Verifying the SCLTN Method. It is apparent in Figure 5(b) that the size of radius selected in log-polar affects the context information of each point in the sequence. Therefore, it is important to select an appropriate radius. To verify the validity of shape context information, it is assumed that the first 10 points of gait acceleration data are selected, and then context information changes of 10 points at different radii are, respectively, obtained, as shown in Figure 9.

As shown in Figure 9, 10\% to $100 \%$ indicate that selected radius length is a percentage of data sequence length. Theoretically, the positions of the first 10 points in a set of gait acceleration data are relatively close and the context information for each point is also similar, so the selected radius should also satisfy the condition. If the difference between adjacent points is large, the selected radius is not suitable. Figure 9 shows the difference in context information between the first 10 points at different radii; according to the variation range of the curve, it is found that the curve changes slightly when the radius is $10 \%$ of the sequence. Therefore, the paper selects $10 \% \times \mathrm{N}$ as the log-polar radius.

We verify the advantages of the SCLTN method over a range of variant methods of Euclidean distance, DTW, and DTW. By calculating the "typical period" proposed in each method in different data sets and the average absolute deviation between its test periods, if the average absolute deviation is small, this indicates that the extracted period is representative and can characterize features of the entire set of gait data and vice versa. 
TABLE 3: Mean and standard deviation of mean absolute deviation between typical cycle and test cycle.

\begin{tabular}{|c|c|c|c|c|c|c|c|c|}
\hline \multirow[b]{2}{*}{ Algorithm } & \multicolumn{2}{|c|}{ OU-Inertial Gait Data } & \multicolumn{2}{|c|}{ SCUT-NAA } & \multicolumn{2}{|c|}{ Data 1 (Xiaomi 4C, 30 people) } & \multicolumn{2}{|c|}{ Data 2 (Samsung Note II, 32 people) } \\
\hline & Mean & Std. & Mean & Std. & Mean & Std. & Mean & Std. \\
\hline Average & 7.08 & 6.86 & 4.31 & 2.42 & 2.54 & 3.08 & 4.06 & 1.67 \\
\hline DTW & 4.85 & 4.14 & 1.12 & 0.39 & 0.84 & 0.28 & 0.83 & 0.75 \\
\hline CRM & 4.67 & 3.99 & 0.79 & 0.27 & 0.51 & 0.20 & 0.64 & 0.79 \\
\hline $\mathrm{CDM}$ & 4.72 & 3.35 & 0.67 & 0.22 & 0.69 & 0.22 & 0.51 & 0.85 \\
\hline Fast-DTW & 5.22 & 6.48 & 2.73 & 3.08 & 1.18 & 0.61 & 1.54 & 0.82 \\
\hline LTN & 4.78 & 4.20 & 0.51 & 0.20 & 0.61 & 0.40 & 0.91 & 0.80 \\
\hline $\mathrm{DTW}+\mathrm{SC}$ & 3.96 & 3.05 & 0.46 & 0.22 & 0.45 & 0.30 & 0.50 & 0.43 \\
\hline $\mathrm{LTN}+\mathrm{SC}$ & 3.89 & 3.46 & 0.43 & 0.26 & 0.43 & 0.13 & 0.48 & 0.30 \\
\hline
\end{tabular}

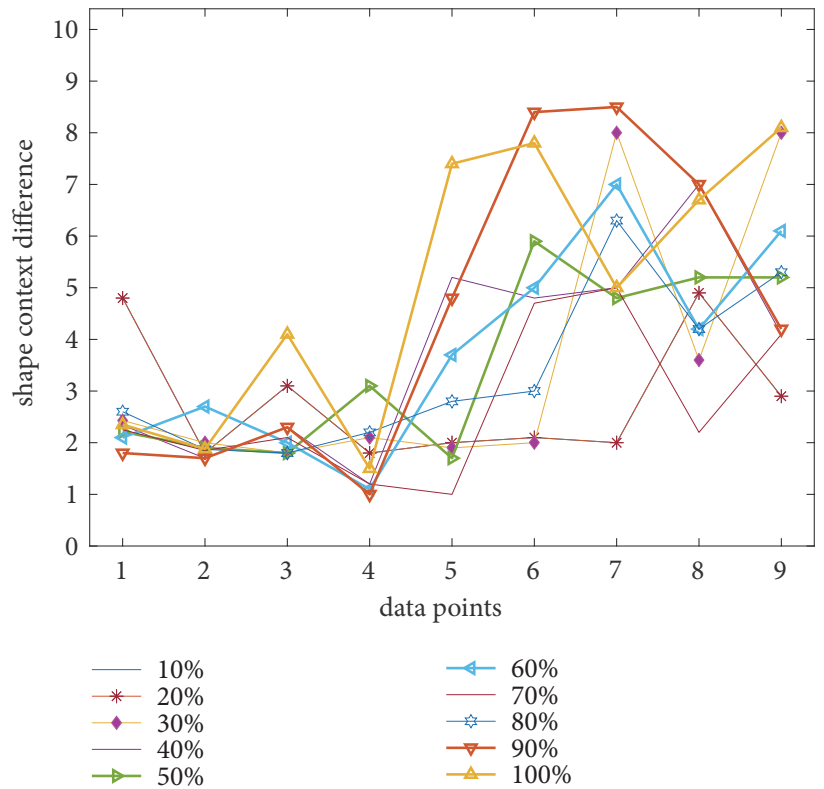

FIgURE 9: Scope of shape change at different radius.

As shown in Table 3, the averaging method normalizes the lengths of all gait sequences and fails to adequately correct misalignment among periods, such that the average absolute deviation is the largest. DTW and its series of deformation algorithms make up for deficiencies in the averaging method, such that it is unnecessary to normalize the length of sequence that is waiting to be compared, and it also effectively solves the time warping problem that is caused by the speed change.

However, DTW is generally used for nonlinear sequence alignment matching, and DTW fails to consider shape features of the sequence itself when matching the alignment. The LTN algorithm is linear and more suitable for the gait data presented in the paper. Compared to the DTW algorithm, the linear relationship between gaits is fully considered and the results are also slightly improved. However, neither DTW nor LTN considers local shape features of gait data or the context information of each point. Therefore, this paper uses the SCLTN algorithm to extract the typical period, from results presented in Table 3 , and it is obvious that the difference value of this part is smaller than those of other results. The SCLTN algorithm considers the linear relationship between gaits, but when the point matching is aligned the local shape at each point is also taken into consideration, and "feature-feature" alignment is realized in the true sense. Hence, a typical period extracted using this method is closer to the original data in terms of numerical value and shape features and is the most representative of the original data features.

7.2.2. Multiclassifier Screening. (a) In Table 1, the following 15 traditional eigenvalues of the single gait period $\mathrm{X}, \mathrm{Y}$, and $\mathrm{Z}$ axes for classification experiments are extracted and listed: mean, standard deviation, skewness, kurtosis, root mean square, over zero rate, interquartile range, period length, energy, and the first 6 Fourier coefficients. Since these are common time-frequency domains and statistical features, the calculation formula is relatively simple and will not be described here.

(b) Sixteen new features, such as the speed variation of relative uniform acceleration motion, are extracted and 


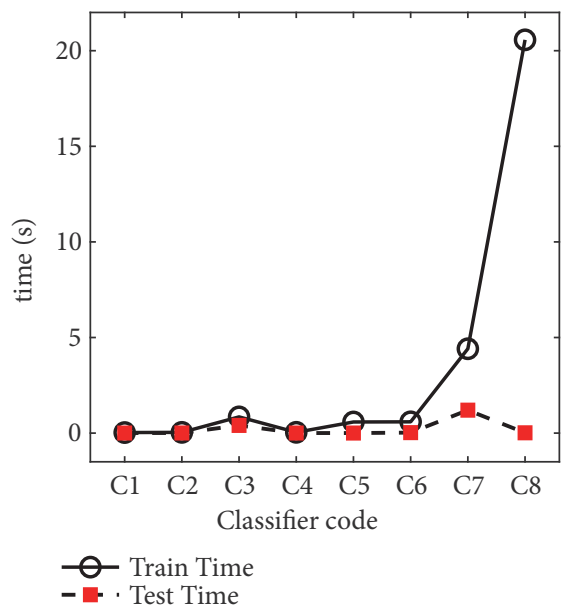

(a) Training test time for traditional feature

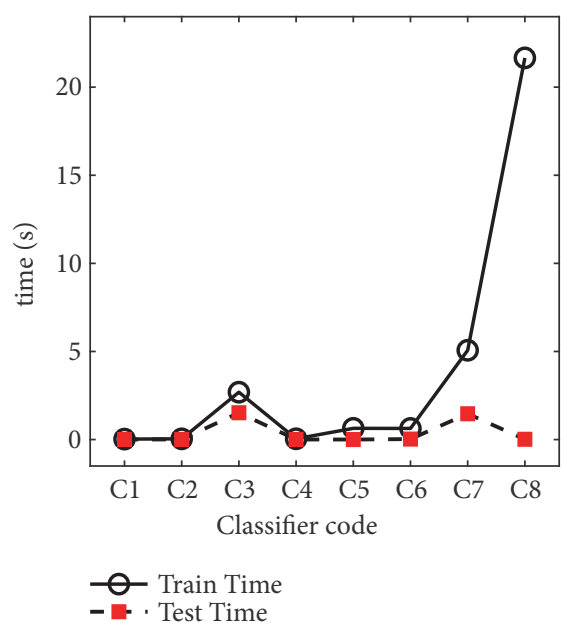

(b) Training test time for new feature

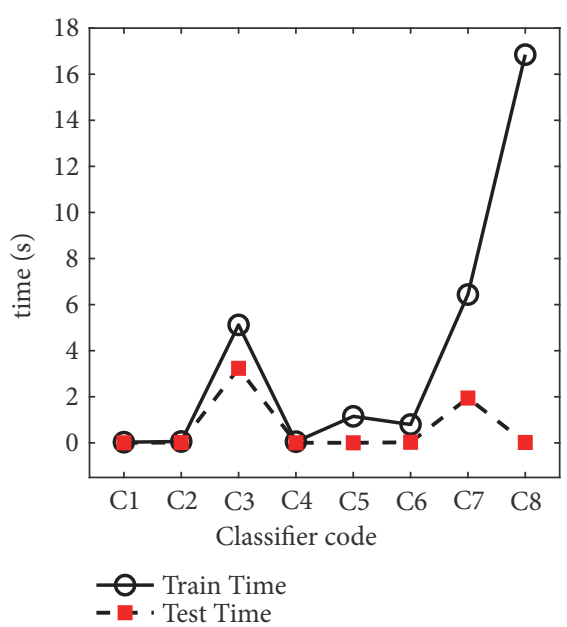

(c) Training test time for fusion feature

FIgURE 10: A training time under different features.

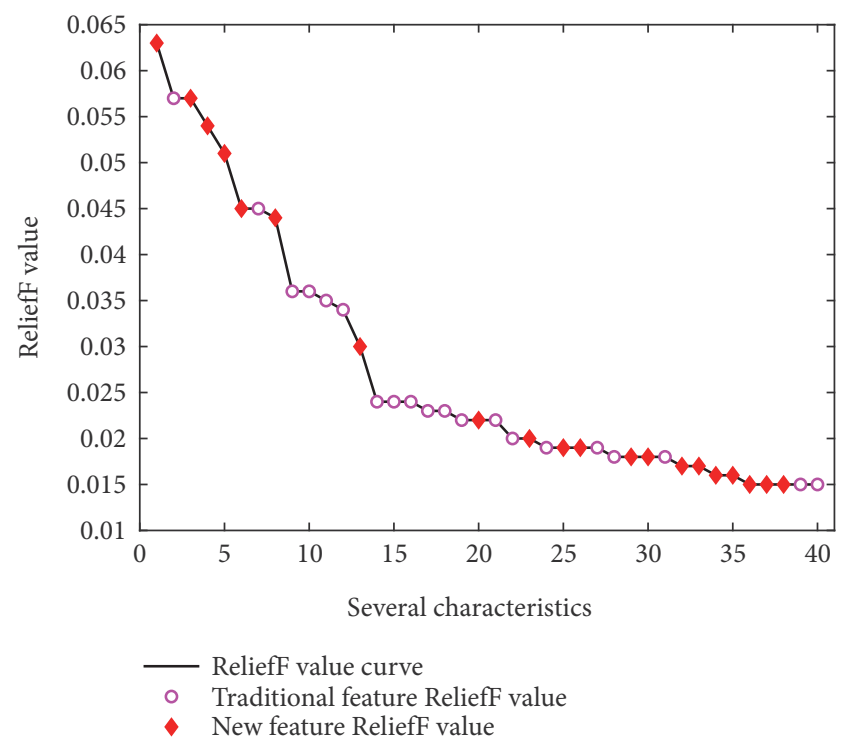

(a) Top 40

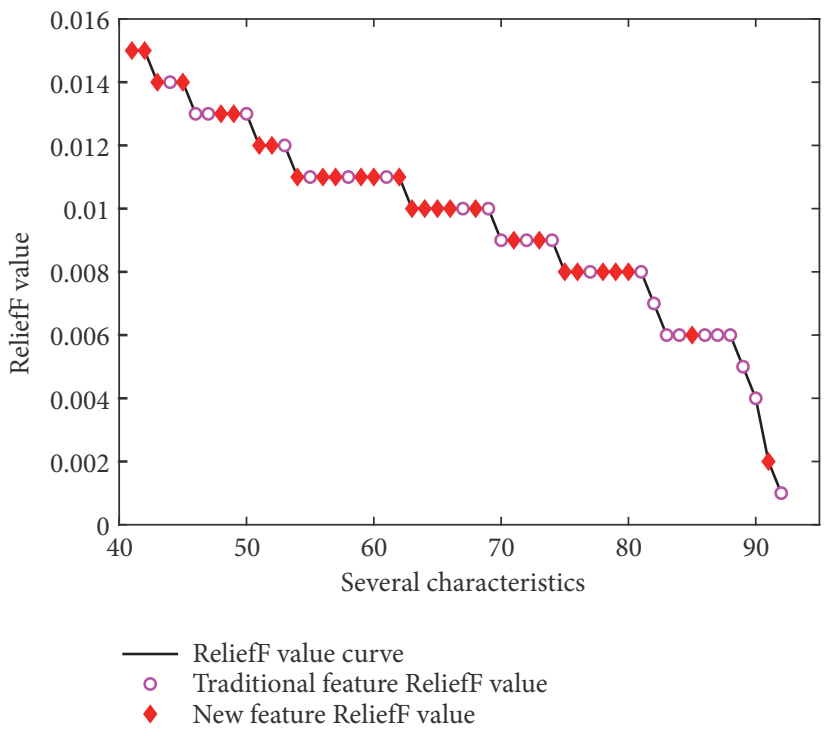

(b) After 53

FIGURE 11: All features correspond to the ReliefF value.

introduced in this paper from the $\mathrm{X}, \mathrm{Y}$, and $\mathrm{Z}$ axes of a single gait period and using the alternative eight classifiers for classification experiments.

(c) Fifteen traditional eigenvalues and 16 new eigenvalues (93 features in total) were extracted and fused for X, Y, and $\mathrm{Z}$ axes of a single gait period. First, the ability of each feature to distinguish samples was calculated using the ReliefF algorithm [34] to verify whether or not the new features have better separability; second, alternative classifiers were used for classification experiments.

It is apparent from Table 4 that the gait data collected in this paper have a good recognition rate for the traditional features on most candidate classifiers, and the highest recognition rate can reach $91.7 \%$. This illustrates that a kind of biometrics, an individual's gait characteristics, can be used for human identification, and traditional gait features have certain separability to gait; however, the recognition rate is not very high.

Let the traditional feature set selected in this paper be T1, the new feature set be $\mathrm{T} 2$, and fusion feature set of new feature and traditional feature be $\mathrm{T} 3$.

Table 4 and Figure 10, respectively, show the test set recognition rate of eight candidate classifiers on three feature sets $\mathrm{T} 1, \mathrm{~T} 2$, and $\mathrm{T} 3$ and the time required to train corresponding classifiers and test set. Figure 11 shows the ReliefF values corresponding to the 93 out-of-synchronization features.

It can be obtained from the second row of Table 4 that gait data collected in this paper has a good recognition rate for the 
TABLE 4: Test set recognition rate.

\begin{tabular}{lcccccrrr}
\hline Classifier & C1 & C2 & C3 & C4 & C5 & C6 & C7 & C8 \\
\hline T1 & 63.47 & $\mathbf{8 2 . 5 7}$ & $\mathbf{8 7 . 6 3}$ & $\mathbf{8 8 . 7 5}$ & $\mathbf{8 6 . 4 2}$ & $\mathbf{9 0 . 1 3}$ & $\mathbf{8 5 . 5 1}$ & $\mathbf{8 0 . 3 4}$ \\
T2 & 54.32 & $\mathbf{6 7 . 2 1}$ & $\mathbf{8 3 . 1 7}$ & $\mathbf{8 8 . 3 5}$ & $\mathbf{7 3 . 0 7}$ & $\mathbf{7 9 . 0 3}$ & $\mathbf{8 4 . 2 3}$ & $\mathbf{7 7 . 0 9}$ \\
T3 & 84.13 & $\mathbf{9 3 . 1 5}$ & $\mathbf{9 3 . 4 5}$ & $\mathbf{9 5 . 4 7}$ & $\mathbf{8 7 . 3 5}$ & $\mathbf{9 3 . 0 5}$ & $\mathbf{8 5 . 4 6}$ & $\mathbf{8 4 . 2 7}$ \\
\hline
\end{tabular}

TABLE 5: Verify the set recognition rate.

\begin{tabular}{lccc}
\hline Classifier & Traditional feature & New feature & Fusion feature \\
\hline C2 & 82.21 & 68.05 & 93.08 \\
C3 & 87.54 & 83.21 & 93.26 \\
C4 & 88.36 & 88.45 & 95.12 \\
C5 & 86.58 & 73.42 & 87.46 \\
C6 & 90.45 & 79.12 & 93.17 \\
\hline
\end{tabular}

traditional features on most of candidate classifiers, and the highest recognition rate can reach $90.13 \%$. This illustrates, as a kind of biometrics, that individual's gait characteristics can be used for human identification, and traditional gait features have certain separability to gait, but the recognition rate is not very high.

It is apparent from the third row of Table 4 that the 16 new eigenvalues extracted in this paper are inferior to the traditional eigenvalues in the classification effect of each classifier, but the gap of recognition rate is not very large. The minimum phase gap is $0.4 \%$; the maximum phase gap is $11.1 \%$; the highest recognition rate of new features is $88.35 \%$, which is $1.78 \%$ of the gap from the highest recognition rate of traditional features. This indicates that the new features have excellent separability and provide a theoretical foundation for subsequent feature fusion.

According to the ReliefF algorithm, the larger the ReliefF value corresponding to a feature, the greater the distinguishing ability of the feature. Figure 11 shows that the new feature has 6 features in the top 10 ReliefF values, the sum of ReliefF values of these 6 new eigenvalues is 0.314 , and the sum of the ReliefF values of the 4 traditional eigenvalues is 0.174 . The new features occupy 20 of the top 40 features of ReliefF values and the sum of the ReliefF values of the 20 new eigenvalues is 0.529; the sum of the ReliefF values of the new eigenvalues is 0.861 among all 93 features, and the sum of the ReliefF values of the traditional eigenvalues is 0.737 . Based on the above analysis, it can be concluded that the new features have better classification effects than traditional features. According to Table 4, compared to the average $6.44 \%$ increase in recognition rate for traditional features, the combination of new features and traditional features has a significant increase in the recognition rate of all candidate classifiers. The highest recognition rate can achieve $95.47 \%$, indicating that feature fusion is very successful.

It can be concluded from Table 4 that eight alternative classifiers obtained better recognition rates whether used in traditional features or new features, or traditional features and new features, which shows that all 8 classifiers have excellent stability. It can be concluded from Figure 10 that classifiers $\mathrm{C} 7$ and $\mathrm{C} 8$ require much training time, and classifiers' time consumption will be greatly integrated if it is used for classifier fusion. Therefore, considering both time consumption and accuracy, C2, C3, C4, C5, and C6 are selected as the base classifiers of the fusion algorithm in this paper.

7.2.3. Verifying the MSV Fusion Algorithm. In this paper, the MSV fusion algorithm is verified in two ways: first by comparing the recognition rate of a verification set under three feature sets and the recognition rate after fusion of the MSV algorithm of a single classifier and second by comparing the recognition rate of the MSV fusion algorithm and different multiclassifier fusion algorithms. Table 5 shows the recognition rates of the five selected base classifiers under three feature sets, and Table 6 shows the comparison experiment results of recognition rates of different fusion algorithms.

It can be concluded from Tables 4 and 5 that the selected medium-based classifiers in Table 5 have better recognition effects in both test set and verification set; the recognition rate is almost the same, showing that the selected base classifier has a good stability.

It can be concluded from Tables 5 and 6, compared to other multiclassifier fusion algorithms, that the recognition rate of three feature sets is improved after the MSV algorithm is merged, and the recognition rate for each feature set is better than those of other fusion algorithms. The recognition rate of three feature sets is $3.30 \%$ higher than that of five base classifiers, and the highest recognition rate is $98.42 \%$ of the fusion feature. From the above analysis, the MSV algorithm is shown to have excellent multiclassifier fusion performance.

7.2.4. Compared with Existing Research. The four related research literatures in recent years are compared with the methods used in this paper to further demonstrate the superiority of the algorithm.

In literature [4], the extracted frequency domain features are classified by the support vector machine (SVM), in which the accuracy rate is $92.7 \%$; the literature [5] uses the support vector machine (SVM) to classify the extracted 
TABLE 6: Recognition rate of different fusion algorithms.

\begin{tabular}{lccc}
\hline Fusion algorithm & Traditional feature & New feature & Fusion feature \\
\hline Traditional voting method & 92.89 & 88.75 & 95.27 \\
Bayes voting [3] & 88.90 & 82.87 & 90.95 \\
MSV algorithm & 94.14 & 91.35 & 98.42 \\
\hline
\end{tabular}

TABLE 7: Comparison with the latest research.

\begin{tabular}{lcccc}
\hline Related literature & Sampling rate & Number of samples & Placement position & Recognition rate \\
\hline Literature [4] & $27 \mathrm{~Hz}$ & 11 & Trouser pocket & $92.70 \%$ \\
Literature [5] & $50 \mathrm{~Hz}$ & 18 & Trouser pocket & $94.0 \%$ \\
Literature [6] & $100 \mathrm{~Hz}$ & 12 & Trouser pocket & $95.0 \%$ \\
Literature [7] & $100 \mathrm{~Hz}$ & 40 & Trouser pocket & $97.10 \%$ \\
This paper & $100 \mathrm{~Hz}$ & 32 & Trouser pocket & $97.78 \%$ \\
\hline
\end{tabular}

frequency domain features and time domain features, in which the accuracy rate is $94.0 \%$; for the extracted frequency domain features and time domain features, the literature [6] selects four classifiers (Random Forest, AdaBoost, K-NN, and Bagging) to vote, in which the accuracy rate is $95.0 \%$; the literature [7] combines trajectory curve features with traditional statistical features, and the accuracy rate obtained by Random Forest is $97.78 \%$.

Combined with Table 7, it is concluded that, whether it is a single frequency domain feature or a combination of time domain features, a better recognition rate can be obtained by optimizing the classifier parameters. Although the literature [6] uses four classifiers, it only uses a simple voting method in the fusion algorithm, which cannot ideally integrate the advantages of each classifiers. The literature [7] not only combines the traditional time domain with frequency domain features but also extracts the corresponding trajectory features from the gait curve to fuse, and its recognition rate is only $0.68 \%$ lower than the paper. However, its time complexity of extracting trajectory features is $O\left(n^{2}\right)$, which is higher than the time complexity of extracting motion features $\left(O\left(\log _{2} n\right)\right)$ in the paper. Based on the above analysis, the gait identification algorithm based on smartphone acceleration data in the paper has definite advantages compared with the existing research.

\section{Conclusion}

The paper improves a method based on LTN, introduces the concept of shape context, proposes a method called SCLTN to extract the typical period from gait data, and adds pointto-point local structure information to enhance LTN in the matching process. This method not only solves the problem of point matching error but also makes all point sets of the gait time series strictly aligned according to point to point. The main advantages are as follows.

(1) The method simultaneously considers differences in values and shapes of a gait sequence.

(2) The method is sensitive to small changes in a time series.
(3) The method gives sufficient consideration to contextual local information for each point in the time series.

In this paper, a single set of gait period data is divided into five motion regions using the maximum and minimum values; the velocity variation of relative uniform acceleration in the motion region is extracted, and the acceleration variation per unit time is taken as a new feature. New features are combined with traditional time domain and frequency domain features to form a new feature set, which can be used to train the five basic classifiers. Prediction results of each classifier are then subjected to the MSV multiclassifier fusion algorithm to obtain a final prediction result.

Experimental results show that the new feature has a good recognition effect for the five base classifiers when using the test set; the highest accuracy rate achieved was $88.35 \%$, and the ReliefF value of the new feature is also higher than the traditional feature value. These results suggest that the new feature has good separability. New features and the traditional feature fusion also achieved a high recognition rate on the test set, and, compared to the single traditional feature recognition rate, the highest recognition rate reached was $95.47 \%$. After the MSV algorithm fusion, the recognition rate increased by $1.34 \%$ on average compared to the maximum recognition rate for the five base classifiers, and the highest recognition rate was $98.42 \%$ for the fusion features. For the feature set that combined new and traditional features, compared to other multiclassifications' fusion algorithms, the MSV fusion algorithm increased by an average of $2.33 \%$.

\section{Data Availability}

The data used to support the findings of this study are available from the corresponding author upon request.

\section{Conflicts of Interest}

The authors declare that there are no conflicts of interest regarding the publication of this paper. 


\section{Acknowledgments}

This study was supported in part by the National Natural Science Foundation of China (Grant No. 61772248) and Zhejiang Public Welfare Technology Research Social Development Project (Grant No. 2017C33223).

\section{References}

[1] L. Rong, Z. Jianzhong, L. Ming et al., "A wearable acceleration sensor system for gait recognition," in Proceedings of the IEEE Conference on Industrial Electronics \& Applications, pp. 26542659, IEEE, May 2007.

[2] M. Muaaz and R. Mayrhofer, "Accelerometer based gait recognition using adapted gaussian mixture models," in Proceedings of the 14th International Conference on Advances in Mobile Computing and Multi Media, pp. 288-291, ACM, New York, NY, USA, 2016.

[3] J. Qu, Z. Zhang, and T. Gong, "A novel intelligent method for mechanical fault diagnosis based on dual-tree complex wavelet packet transform and multiple classifier fusion," Neurocomputing, vol. 171, no. 1, pp. 837-853, 2016.

[4] H. M. Thang, V. Q. Viet, N. D. Thuc, and D. Choi, "Gait identification using accelerometer on mobile phone," in Proceedings of the 2012 International Conference on Control, Automation and Information Sciences, ICCAIS 2012, pp. 344-348, IEEE, 2012.

[5] H. W. Yang and J. M. Yang, "Gait recognition based on accelerometer of mobile phone," Journal of Nanyang Institute of Technology, vol. 4, pp. 20-23, 2017.

[6] H. Donggu, Human Gait Recognition and Application Based on Smart-Phone, Research Institute of Electronic Science and Technology, 2018.

[7] Y. C. Wan, Gait Identification Based on Mobile-Phone Acceleration Sensor, ChangZhou University, 2018.

[8] M. O. Derawi, P. Bours, and K. Holien, "Improved period detection for accelerometer based gait authentication," in Proceedings of the International Conference on Intelligent Information Hiding \& Multimedia Signal Processing, 2010.

[9] B. Sun, Y. Wang, and J. Banda, "Gait characteristic analysis and identification based on the iPhone's accelerometer and gyrometer," Sensors, vol. 14, no. 9, pp. 17037-17054, 2014.

[10] L. N. Zhang, Gait Feature Authentication Based on Acceleration Sensor, Shenyang University of Technology, 2014.

[11] M. Muaaz and R. Mayrhofer, "An analysis of different approaches to gait recognition using cell phone based accelerometers," in Proceedings of the International Conference on Advances in Mobile Computing \& Multimedia, p. 293, ACM, New York, NY, USA, 2013.

[12] W. Zhongmin, W. Ke, and E. H. Yan, "Multiple classifier fusion model for activity recognition based on high reliability weighted," Journal of Computer Applications, vol. 36, no. 12, pp. 3353-3357, 2016.

[13] Y. Yuan, C. Wang, J. Zhang, J. Xu, and M. Li, "An ensemble approach for activity recognition with accelerometer in mobile-phone," in Proceedings of the International Conference on Computational Science and Engineering, pp. 1469-1474, IEEE Computer Society, 2014.

[14] A. Bayat, M. Pomplun, and D. A. Tran, "A study on human activity recognition using accelerometer data from smartphones," Procedia Computer Science, vol. 34, pp. 450-457, 2014.
[15] S. Sprager and M. B. Juric, "An efficient HOS-based gait authentication of accelerometer data," IEEE Transactions on Information Forensics and Security, vol. 10, no. 7, pp. 1486-1498, 2015.

[16] H. Lu, J. Huang, T. Saha, and L. Nachman, "Unobtrusive gait verification for mobile phones," in Proceedings of the 2014 ACM International Symposium on Wearable Computers, pp. 91-98, ACM, New York, NY, USA, 2014.

[17] M. Derawi and P. Bours, "Gait and activity recognition using commercial phones," Computers \& Security, vol. 39, pp. 137-144, 2013.

[18] Z. Lv, X. Xing, K. Wang, and D. Guan, "Class energy image analysis for video sensor-based gait recognition: a review," Sensors, vol. 15, no. 1, pp. 932-964, 2015.

[19] T. T. Ngo, Y. Makihara, H. Nagahara, Y. Mukaigawa, and Y. Yagi, "Orientation-compensative signal registration for owner authentication using an accelerometer," IEICE Transaction on Information and Systems, vol. E97-D, no. 3, pp. 541-553, 2014.

[20] R. Tavenard and L. Amsaleg, "Improving the efficiency of traditional DTW accelerators," Knowledge and Information Systems, vol. 42, no. 1, pp. 215-243, 2015.

[21] X. Ruan and C. Tian, "Dynamic gesture recognition based on improved DTW algorithm," in Proceedings of the 12th IEEE International Conference on Mechatronics and Automation, ICMA 2015, pp. 2134-2138, Beijing, China, August 2015.

[22] M. M. Ardestani and M. A. Wimmer, "Can a linear combination of gait principal component vectors identify hip OA stages?" Journal of Biomechanics, vol. 49, no. 10, pp. 2023-2030, 2016.

[23] S. Farr, A. Kranzl, J. Hahne, and R. Ganger, "Rotational gait patterns in children and adolescents following tension band plating of idiopathic genua valga," Journal of Orthopaedic Research, vol. 35, no. 8, pp. 1617-1624, 2017.

[24] S. Yuzhe, L. Shanzhen, and L. Shaobin, "License plate character recognition research based on shape context," in Proceedings of the 4th International Conference on Instrumentation and Measurement, Computer, Communication and Control, IMCCC 2014, pp. 489-492, September 2014.

[25] C. Li and S. Qian, "Measuring image similarity based on shape context," International Journal of Multimedia and Ubiquitous Engineering, vol. 10, no. 3, pp. 127-134, 2015.

[26] W. Fang and J. Li, "A morphing of linear feature based on shape context matching," Geomatics and Information Science of Wuhan University, vol. 42, no. 7, pp. 963-967, 2017.

[27] P. N. Narwade, R. R. Sawant, and S. V. Bonde, "Offline handwritten signature verification using cylindrical shape context," 3D Research, vol. 9, no. 4, p. 48, 2018.

[28] Y. Liu, K. Sun, and H. Xie, "High accurate contour vision algorithm based on shape context and ICP," Journal of South China University of Technology (Natural Science), vol. 46, no. 1, pp. 131-144, 2018.

[29] Y. Watanabe, "Influence of holding smart phone for acceleration-based gait authentication," in Proceedings of the 5th International Conference on Emerging Security Technologies, EST 2014, pp. 30-33, Spain, September 2014.

[30] T. Hoang and D. Choi, "Secure and privacy enhanced gait authentication on smart phone," The Scientific World Journal, vol. 2014, Article ID 438254, 8 pages, 2014.

[31] A. Primo, V. V. Phoha, R. Kumar, and A. Serwadda, "Contextaware active authentication using smartphone accelerometer measurements," in Proceedings of the 2014 IEEE Conference on Computer Vision and Pattern Recognition Workshops, CVPRW 2014, pp. 98-105, June 2014. 
[32] Q. Jing-Guang, W. Y. Song, E. Y. Qiang et al., "The biomechanics principle of walking and analysis on gaits," Journal of Nanjing Institute of Physical Education, vol. 5, no. 4, pp. 1-7, 2006.

[33] K. Wang, Application and Research of Multiple Classifier Fusion Algorithm in Activity Recognition, Xian university of Posts \& Telecommunications, 2017.

[34] L. Huang, J. Tang, D. Sun, and B. Luo, "Feature selection algorithm based on multi-label ReliefF," Journal of Computer Applications, vol. 32, no. 10, pp. 2888-2898, 2012. 


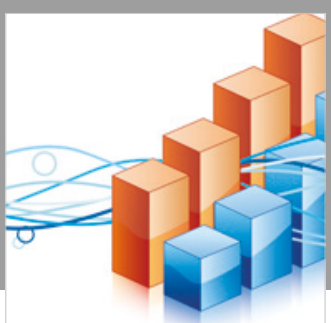

Advances in

Operations Research

\section{-n-m}
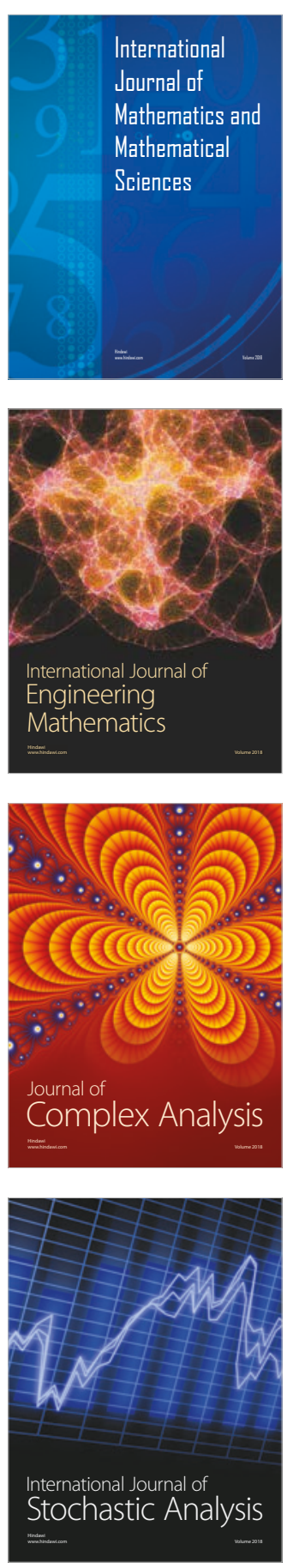
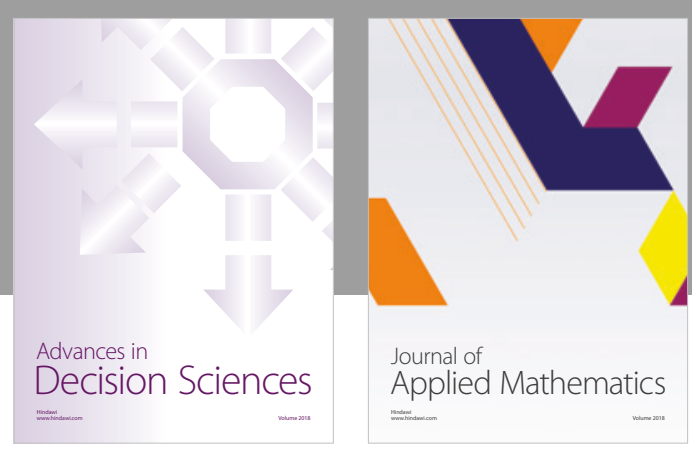

Journal of

Applied Mathematics
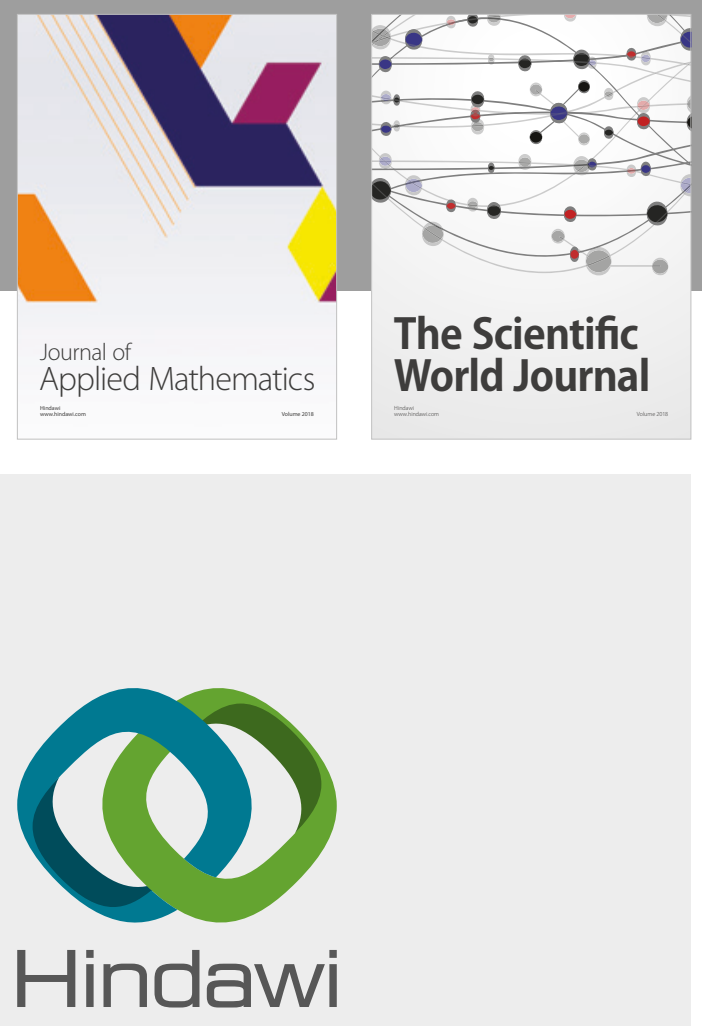

Submit your manuscripts at

www.hindawi.com

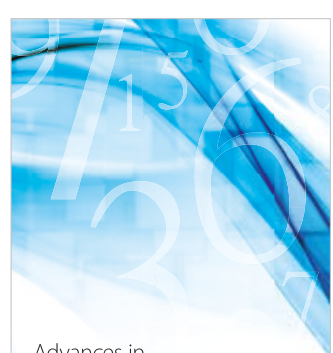

Advances in
Numerical Analysis
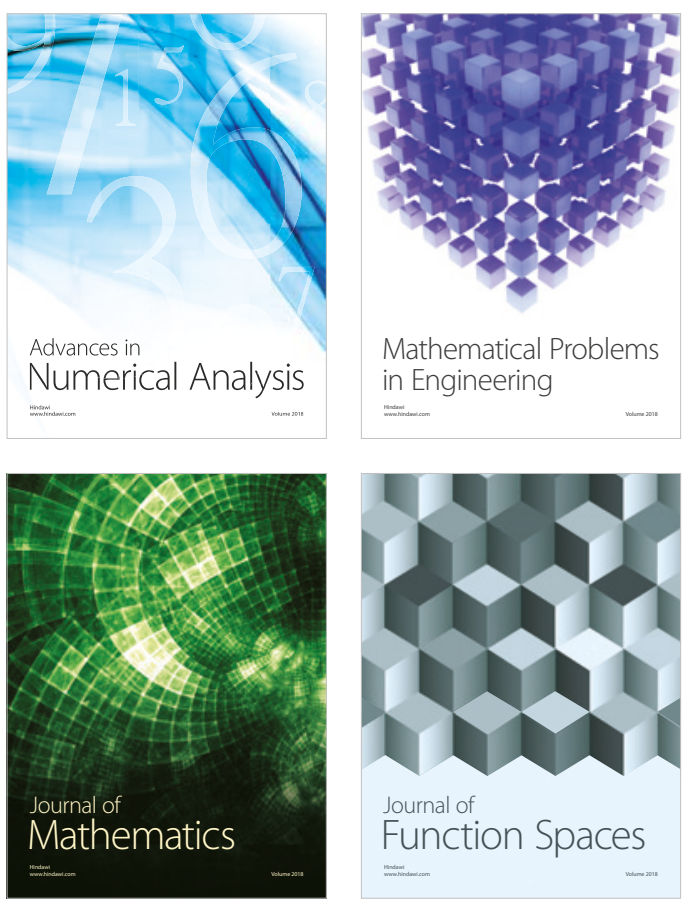

Mathematical Problems in Engineering

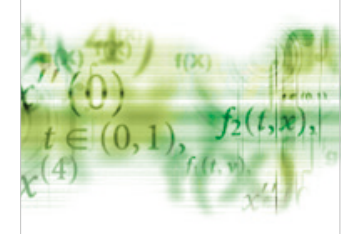

International Journal of

Differential Equations

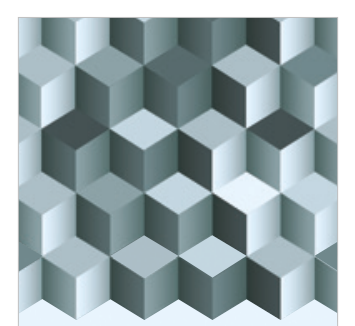

Journal of

Function Spaces
The Scientific

World Journal

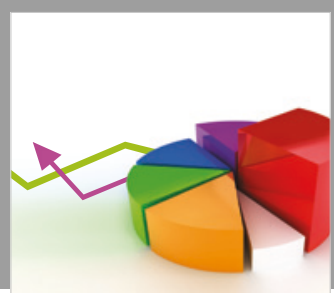

Journal of

Probability and Statistics
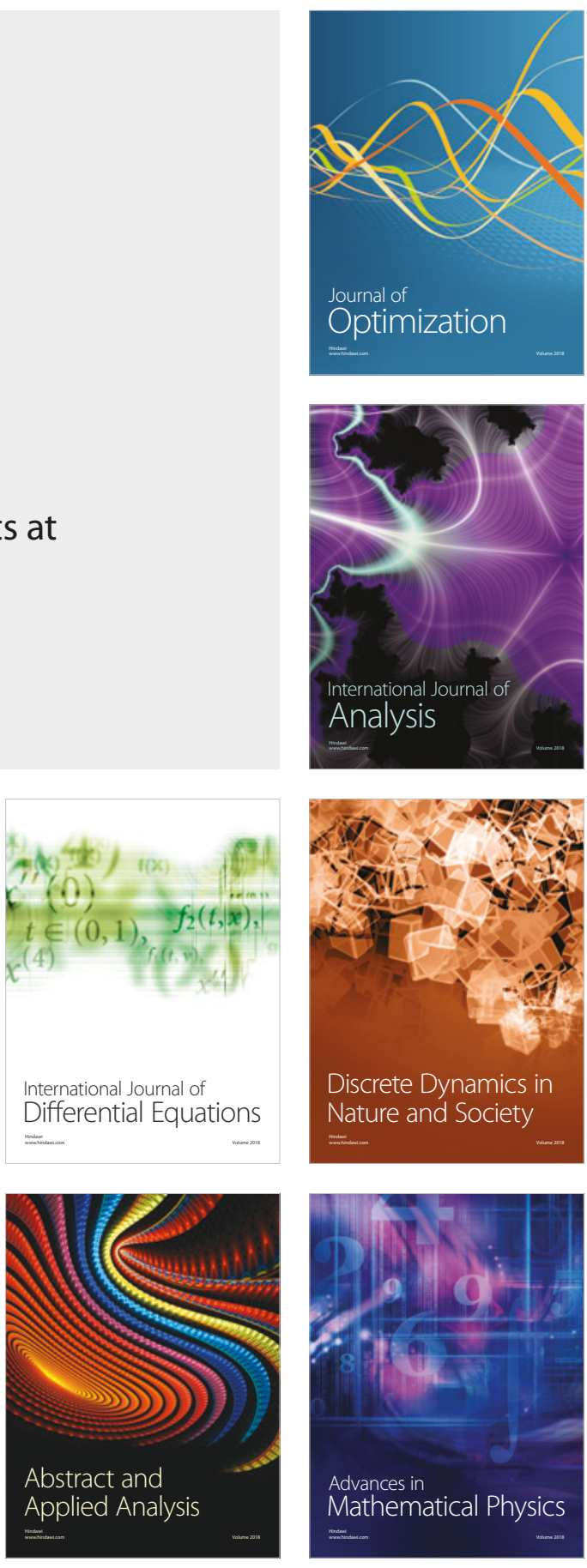